\title{
Active and passive critical slip fields for cohesionless soils and calculation of lateral earth pressures
}

\author{
D.-Y. ZHU*, Q.-H. QIAN* and C. F. LEE†
}

\begin{abstract}
A new method of solving earth pressure problems is proposed in this paper within the framework of the limit equilibrium approach. The concept of the critical slip field (CSF) is postulated: the active critical slip field (ACSF) in the active case, and the passive critical slip field (PCSF) in the passive case. Based on the principle of extremum thrust force (which is theoretically consistent with the principle of optimality) in conjunction with the method of slices, a numerical procedure is presented for the determination of such fields and consequently the distribution of earth pressures on retaining walls. For simplicity at this stage, the backfill material is assumed to be a homogeneous cohesionless soil with sloping ground surface carrying uniform and vertical surcharge, while the retaining wall is allowed to be battered, and the strength of the soil may be either fully or partially mobilised. Compared with the rigorous method (i.e. the method of characteristics), the proposed method is simple in principle and easily implemented in a computer program. However, it is more accurate than other approximate techniques and agrees well with available closed-form solutions. A number of examples of ACSF and PCSF are given in this paper, and the validity and efficiency of the proposed method are demonstrated. In addition, it is easy to extend this numerical procedure to obtain more general ACSF and PCSF accommodating non-homogeneous $c, \phi$ soils subject to complicated loading conditions.
\end{abstract}

KEYWORDS: earth pressure; failure; numerical modelling and analysis; retaining walls.
Nous proposons dans cet exposé une nouvelle méthode pour résoudre les problèmes de pression de contact en utilisant la méthode d'équilibre limite. Nous prenons le concept d'un champ de glissement critique (CSF) et nous étudions le champ de glissement critique actif (ACSF) dans le cas actif et le champ de glissement critique passif (PCSF) dans le cas passif. En nous basant sur le principe de force de poussée extrême (qui est théoriquement en accord avec le principe d'optimalité) en conjonction avec la méthode de tranches, nous présentons un procédé numérique permettant de déterminer ce type de champ et, de là, la répartition des pressions de contact sur les murs de soutènement. Pour simplifier à ce stade, nous supposons que la matière de remblayage est un sol homogène non cohésif avec une surface inclinée portant une surcharge uniforme et verticale, que le mur de soutènement est martelé et que la résistance du sol est soit entièrement soit partiellement mobilisée. Par rapport à la méthode rigoureuse (c'est-à-dire la méthode des caractéristiques), la méthode proposée est de principe simple et elle est facile à mettre en uvre dans un programme informatique. Par ailleurs, elle est plus exacte que d'autres techniques approximatives et s'accorde bien avec les solutions de forme fermée qui sont disponibles. Nous donnons dans cet exposé plusieurs exemples de ACSF et de PCSF et nous démontrons la validité et l'efficacité de la méthode proposée. De plus, il est facile d'étendre ce procédé numérique pour obtenir des ACSF et PCSF plus généraux, tenant compte d'un sol $\varnothing, c$ non homogène, soumis à des conditions de charge compliquées.

\section{INTRODUCTION}

The determination of earth pressure plays a primary role in the design of retaining walls, sheet piles, and other geotechnical structures. The earth pressure theories of Coulomb and Rankine (Terzaghi, 1943) still occupy a dominant place in geotechnical engineering practice, though more sophisticated methods have been developed in recent decades. The Rankine theory is rigorous but limited to simple situations, while the Coulomb theory is more versatile in accommodating complex configurations of backfills and loading conditions, as well as frictional effects between walls and backfills. The assumption underlying the Coulomb theory is that a planar failure surface is developed in the backfill, and the lateral earth force against the wall is determined by satisfying force equilibrium. However, both theoretical and experimental studies have shown that this assumption is not perfectly valid when the wall is rough, especially in the passive case, which often leads to non-conservative estimations of earth pressures.

More than 50 years ago, several researchers dealt with the earth pressure problem using curved failure surfaces on the basis of the limit equilibrium principle (Krey, 1936; Terzaghi, 1943; Caquot \& Kerisel, 1948). The failure surface was usually

Manuscript received 2 September 1998; revised manuscript accepted 8 February 2001

Discussion on this paper closes 2 November 2001, for further details see the inside back cover.

* Nanjing Engineering Institute, China.

$\uparrow$ University of Hong Kong. assumed to be a circle, an ellipse, a log-spiral, or a combination of a log-spiral and a straight line. However, the process of such calculations was cumbersome in practical application, and the accuracy was not warranted. Some attempts have been made to use plasticity theory for obtaining earth pressures since the method of characteristics was introduced in soil stability analysis (Sokolovski, 1965; Graham, 1968; Lee \& Herington, 1972; Hettiarachi \& Reece, 1974). Closed-form solutions based on plasticity theory are few, and numerical procedures must be involved in obtaining the slip line in the backfill. Because of its inefficiency in dealing with complex boundary conditions, the slip-line method cannot be easily incorporated into a general computer program and is therefore limited in practical application. In the last two decades, limit analysis has been used to obtain upper bound solutions for passive earth pressure problems and lower bound solutions for active ones (Chen, 1975; Chen \& Liu, 1990), but it is not an easy task to choose an appropriate kinematically admissible mechanism.

The method of slices, as commonly used in slope stability analysis, was first employed for the determination of lateral earth forces by Janbu (1957). However, only a few investigators made further attempts to enhance its application in earth pressure studies (Shields \& Tolunay, 1973; Rahardio \& Fredlund, 1983). In spite of its adaptability to complex loading and geometrical conditions, the method of slices has two inherent weaknesses. First, assumptions would have to be made regarding the interslice force to render the problem determinate, and different assumptions might lead to different values of lateral earth forces. Second, the shape of the potential failure surface has to be specified before searching for the critical one. Because of these drawbacks, the solution for lateral earth force 
by the conventional method of slices deviates considerably from other solutions, and cannot be reliably applied to engineering practice.

In this paper, the authors propose a new effective method for determining earth pressures as well as critical slip fields in cohesionless soils within the framework of the limit equilibrium method of slices.

\section{FUNDAMENTALS OF METHOD OF SLICES}

In accordance with most earth pressure theories, a sliding mass develops when the soil-wall system is on the verge of limiting equilibrium, with strengths along the failure surface assumed to be fully mobilised or partly mobilised to a specified degree. At this stage, the position of the failure surface is prescribed.

Consider a typical soil-wall system as shown in Fig. 1, where the wall back is inclined at angle $\omega$ to the vertical and the slope of the backfill surface is $\beta$. $\omega$ is positive when the wall is inclined into the backfill, and $\beta$ is also positive when the backfill surface moves upwards from the top of the wall. A uniform vertical surcharge of density $q$ in the horizontal plane is distributed along the backfill surface. The wall height is $H$ and its oblique height $L$ is equal to $H / \cos \omega$. The internal friction angle of the soil is $\phi$ and the unit weight is $\gamma$. The wall friction angle is $\delta$. Following the sign convention in the literature, $\delta$ is positive when the wall moves upwards relative to the soil in the active case, or when it moves downwards relative to the soil in the passive case. In order to simplify derivations, nominal angles of soil friction and wall friction are introduced herein, labelled as $\bar{\phi}$ and $\bar{\delta}$ respectively, where $\phi=\phi, \delta=\delta$ for the active case, and $\bar{\phi}=-\phi, \bar{\delta}=-\delta$ for the passive case. Thus most of the equations involved that correspond to both the active and passive cases can be incorporated into generalised ones. In other words, we can regard the passive case as an alternative active case with the signs of the soil and wall friction changed, but the nature of the extremum of the lateral force still remains: that is, maximum (minimum) for the active (passive) case.

Specifying a reference coordinate system as indicated in Fig. 1 , the sliding mass above the failure surface is divided into $n$ slices parallel to the $y$-axis or the back of the wall. The interslice force (also called the side force) between the $k$ th slice and its lower one is denoted as $Z_{k}$. It is apparent that $Z_{0}=0$ at the upper end and $Z_{n}=P$ at the wall, where $P$ is the lateral force acting on the wall, labelled as $P_{\mathrm{a}}$ in the active case and $P_{\mathrm{p}}$ in the passive case. The point of application of $P$ is known in some cases and is unknown in others. Herein, the ratio of the height of such a point above the wall toe to the wall height, $H$, is assumed to be $\rho$, which is either specified beforehand or determined later in the process of solution. Theoretically, almost all the methods of slices may be used for calculating lateral forces, but only the Morgenstern-Price method (Morgenstern \& Price, 1965) has been adopted for the present problem because it satisfies the conditions for both force and moment equilibrium, and involves fewer numerical difficulties than other methods. In this method, an assumption is made with interslice forces to render the problem determinate. A function (called the interslice force function) was used, including a scaling parameter to describe the ratio of the normal to tangential interslice force across the sliding mass. In other words, the directions of interslice forces are represented by a function. The lateral force and the scaling parameters are obtained by satisfying the conditions for both force and overall moment equilibrium. For earth pressure problems, four types of interslice force functions (FUN1, 2, 3 and 4, each involving a parameter, $m$, to be determined) have been suggested, which also take into account the theoretical conditions for certain boundaries (see details in Appendix 1).

By investigating the force equilibrium for individual slice and moment equilibrium for the whole sliding mass, recurrence equations of interslice forces $\left(Z_{k}\right)$ and moments $\left(M_{k}\right)$ can be derived as follows (the derivation is given in Appendix 2):

$$
\begin{aligned}
Z_{k}= & \frac{1}{\cos \left(\theta_{k}-\alpha_{k}+\bar{\phi}\right)}\left[Z_{k-1} \cos \left(\theta_{k-1}-\alpha_{k}+\bar{\phi}\right)\right. \\
& \left.+\left(Q_{k}+W_{k}\right) \sin \left(\alpha_{k}+\omega-\bar{\phi}\right)\right] \\
M_{k}= & M_{k-1}+\left[\frac{\sin \left(\alpha_{k}-\theta_{k}\right)}{\cos \alpha_{k}} \cdot \frac{b_{k}}{2}+\frac{\sin \left(\alpha_{k+1}-\theta_{k}\right)}{\cos \alpha_{k+1}} \cdot \frac{b_{k+1}}{2}\right] \cdot Z_{k} \\
& +W_{k} \sin \omega \frac{h_{k}}{2}+Q_{k} \sin \omega \cdot h_{k}
\end{aligned}
$$

where the notations are as given in Appendix 2.

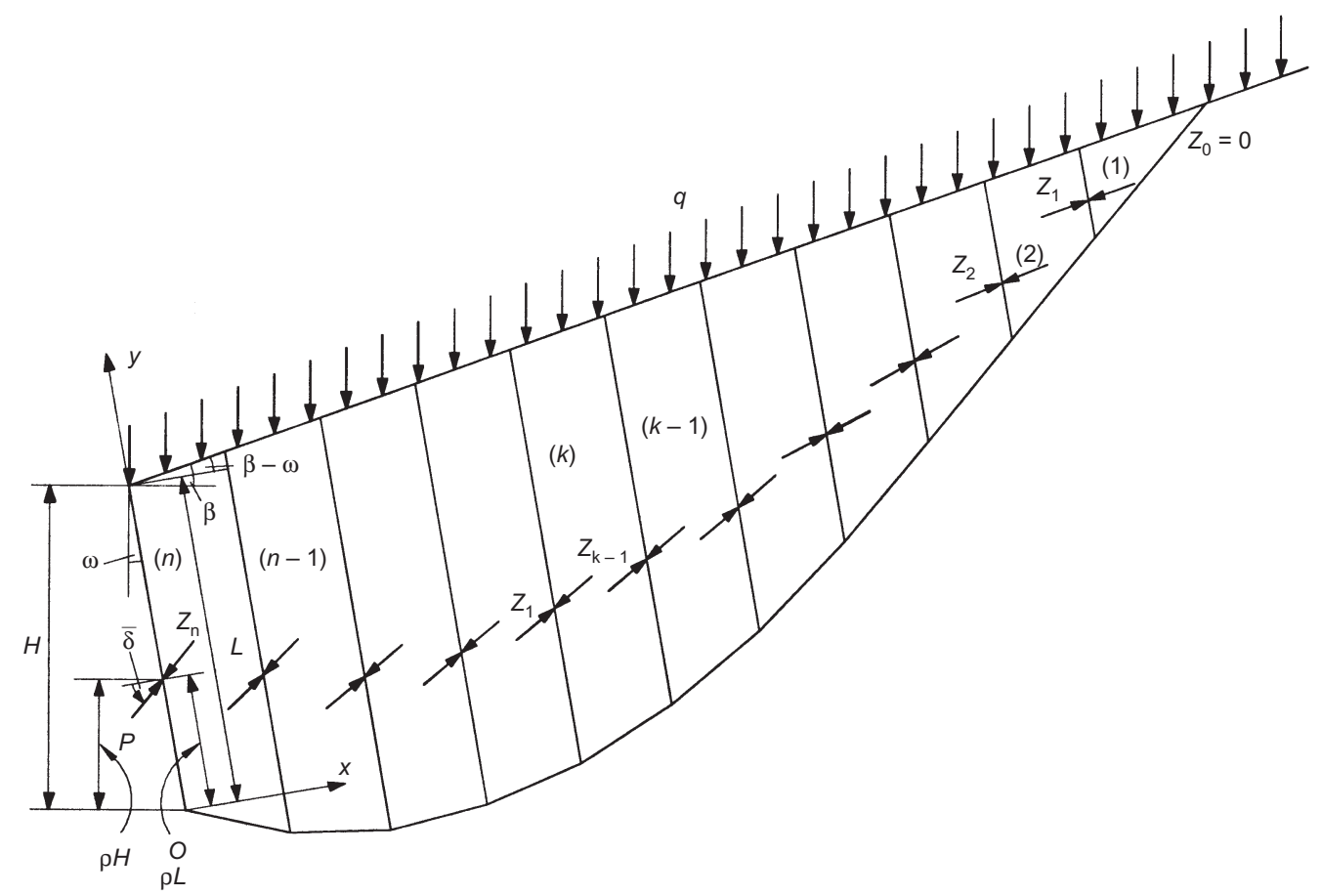

Fig. 1. Division of sliding mass 
Taking moments about $\mathrm{O}$ of the lateral force $P\left(=Z_{n}\right)$ and considering the overall moment equilibrium for the sliding mass, we have

$$
M_{n}-Z_{n} \cdot \rho \cdot L \cdot \cos \delta=0
$$

A more general moment equilibrium equation may be in the form

$$
M_{n}+C_{1} Z_{n}+C_{2}=0
$$

where $C_{1}$ and $C_{2}$ are constants that relate to the nature of the problem under consideration.

For a given failure surface, selecting an interslice force function with an assumed value of parameter $m$, the lateral force $P$ can be obtained by using equation (1) in an iterative manner. It is necessary to check the moment equilibrium condition (equation (4)) ( $M_{n}$ is obtained by using equation (2) in the same manner). If such a condition is not satisfied, then another value of the parameter $m$ should be used. This process is repeated until the moment equilibrium condition is satisfied within a specified tolerance. Generally, this trial-and-error procedure is tedious and inefficient. Therefore, a more effective technique based on the Newton-Raphson method has been developed. This is given in detail in Appendix 3.

\section{POSTULATION OF CSF AND PRINCIPLE OF EXTREMUM THRUST} FORCE

\section{Postulation of CSF}

The concept of slip fields in the soil began many years ago in the theory of plasticity (Sokolovski, 1965). According to this theory, two families of conjugate slip lines develop within the plastic region, which are characteristic of stress or velocity. The stress at any point in the slip-line field satisfies a force equilibrium and a failure criterion. Experiments also confirmed the existence of such a field in the backfill behind a retaining wall (James \& Bransby, 1970). With this background, it is justifiable to postulate that a family of slip surfaces exists in the backfill, along which the sliding masses produce pressures against the wall.

Figure 2(a) presents the schematic pattern of a slip field and associated distribution of earth pressures $p(z)$ along the wall. Each slip surface corresponds to a sliding mass that provides a lateral force $P(z)$, as shown in Fig. 2(b). The resultant of surcharge $Q(\mathrm{z})$, the weight $W(\mathrm{z})$ and the resistance force $R(z)$ for the sliding mass produce a total moment $M(\mathrm{z})$ about $\mathrm{A}$. The relationships between $p(z), P(z)$ and $M(z)$ are as follows:

$$
P(z)=\int_{0}^{z} p(\varsigma) \mathrm{d} \varsigma
$$

$$
M(z)=\cos \delta \cdot \int_{0}^{z} p(\varsigma)(z-\varsigma) \mathrm{d} \varsigma
$$

If the slip field is specified, then the lateral force for each surface can be calculated by using the method of slices, with the moment equilibrium condition (equation (6)) satisfied. Since the soil is at the active or passive limiting state, the slip surface should be such that $P(z)$ attains a maximum value in the active state or a minimum value in the passive state. In this situation, the slip surface is referred to as the critical slip surface. At any given depth of the wall, there may be a critical slip surface that yields a maximum (or minimum) lateral force in the active (or passive) state. Then, all of these critical slip surfaces will constitute a field that is defined as the critical slip field, or CSF. A CSF is redefined as an ACSF (active critical slip field) in the active case or a PCSF (passive critical slip field) in the passive case.

Note that the CSF should not be identified with the slip-line field. The latter is based on rigorous plasticity theory, and requires satisfaction of force equilibrium and failure criterion at any point in the field. As for the CSF, it only satisfies the force and moment equilibrium conditions for sliding masses corresponding to a series of critical slip surfaces, and takes into account the failure condition along these surfaces. Thus the CSF is still within the framework of the rigorous limit equilibrium approach. As will be discovered, especially in some special cases where closed-form solutions of slip-line fields are available, the CSF coincides approximately with the theoretical field (their solutions of earth pressures are nearly the same) though their backgrounds are essentially different.

\section{Principle of extremum thrust force}

As is already known, the critical slip surface in the CSF, say line $\mathrm{AC}$ in Fig. 3(a), should yield a maximum or minimum lateral force, say $P_{\mathrm{A}}$, at the wall in the active or passive state. In other words, for a fixed exit point at the wall, any surface deviating from the critical one will give lateral forces of magnitude smaller or larger than $P_{\mathrm{A}}$ in the active or passive state.

Choose an arbitrary point, B in Fig. 3(a), on line AC and visualise a plane through $B$ and parallel to the wall back as an interface of a slice. The resultant force acting on that face from $\mathrm{B}$ to the ground surface is $P_{\mathrm{B}}$, whose inclination is assumed to be specified. It is obvious that the magnitude of $P_{\mathrm{B}}$ is dependent only on the location of line $\mathrm{BC}$, and is independent of line $\mathrm{BA}$. The potential surfaces through $\mathrm{B}$ to the ground surface are many, for example $\mathrm{BC}_{1}, \mathrm{BC}_{2}, \mathrm{BC}_{3}$, among which the critical surface (BC) should still cause the lateral force $P_{\mathrm{A}}$ to be a maximum (or minimum) in the active (or passive) state. It can be seen from the recurrence relation between interslice forces
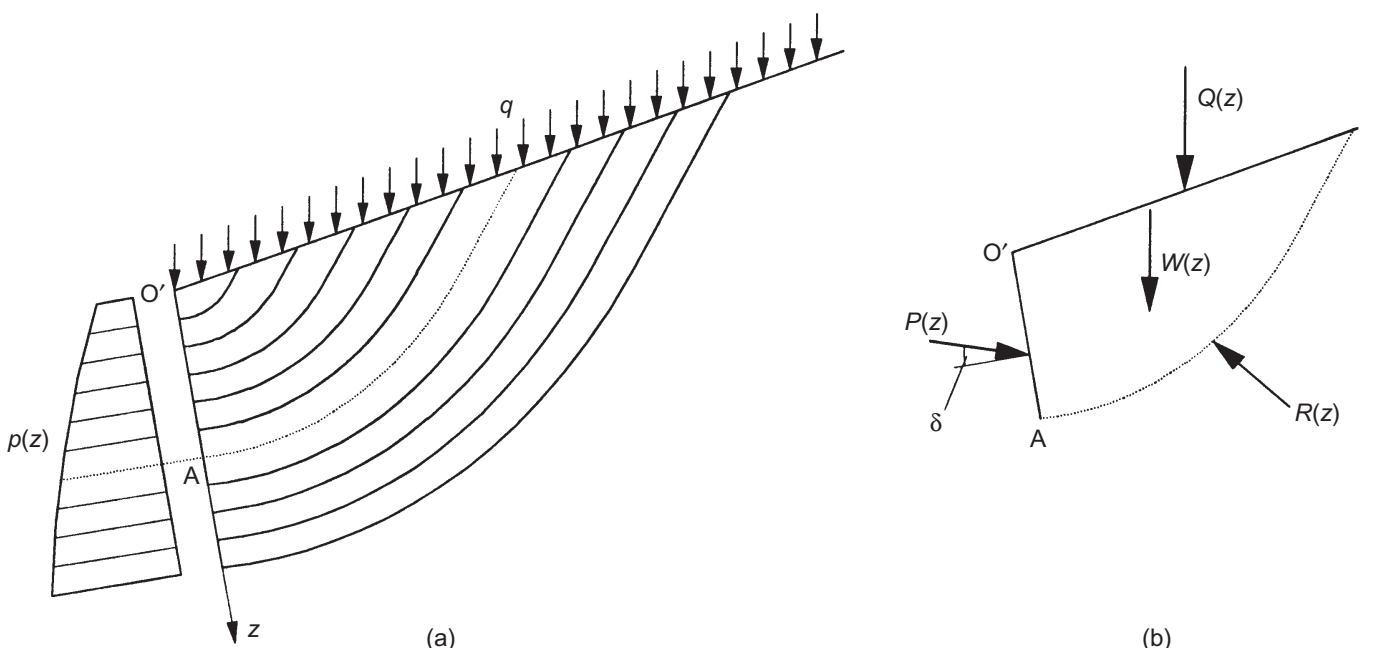

Fig. 2. Postulation of critical slip field (CSF): (a) schematic pattern of CSF; (b) forces on a typical sliding mass 

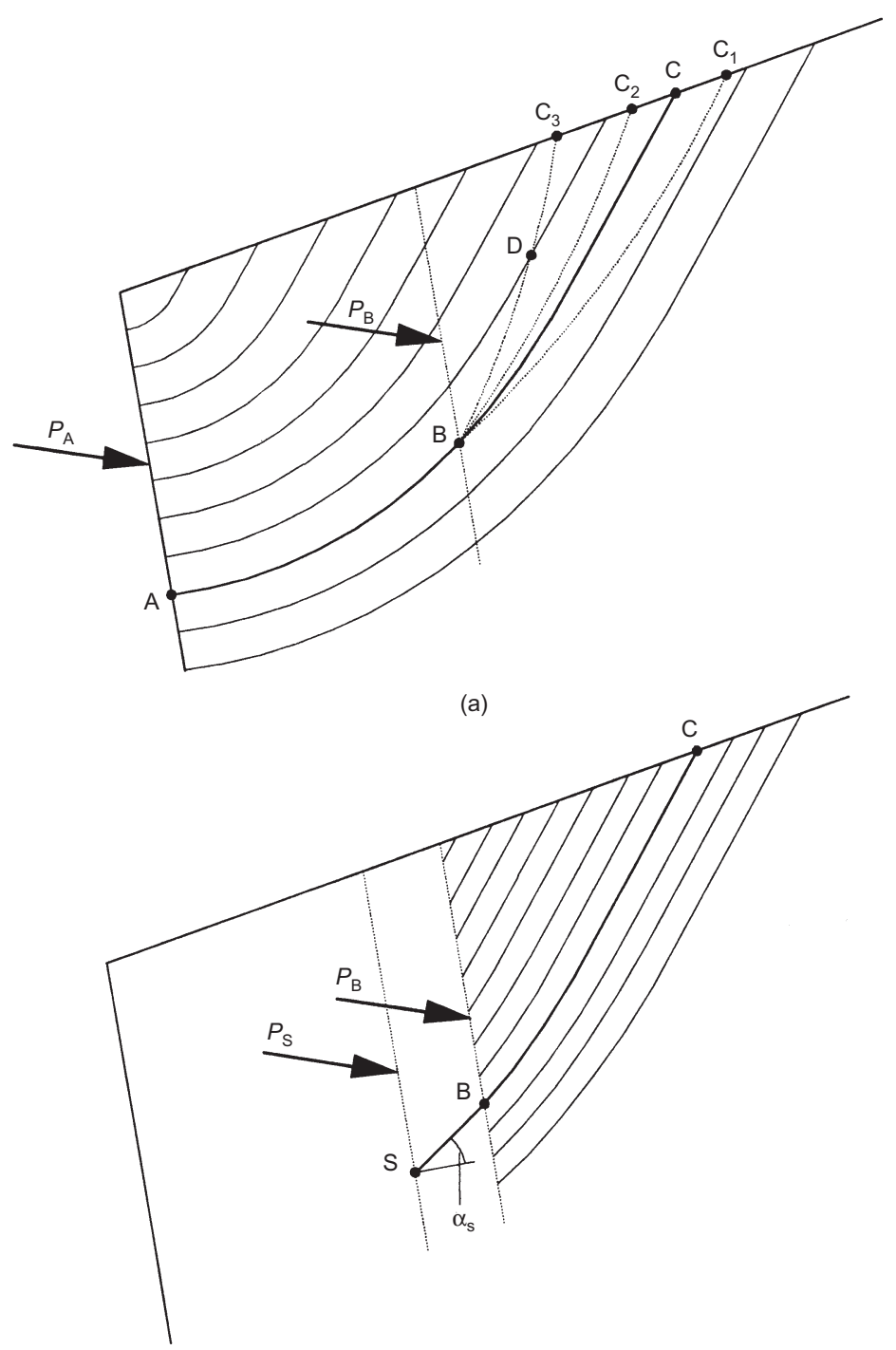

(b)

Fig. 3. Illustration of the principle of extremum thrust force: (a) characteristic of a critical slip surface; (b) critical slip direction and critical thrust force

(equation (1)) that, in order for the lateral force $P_{\mathrm{A}}$ to attain its maximum (or minimum) value in the active (or passive) case, the thrust force $P_{\mathrm{B}}$ should also be a maximum (or minimum), or in general an extremum. This force is defined as the critical thrust force for point $\mathrm{B}$, and this is referred to as the principle of extremum thrust force. This principle implies that, for any critical slip surface passing from the wall to the ground surface, any arbitrary part of the surface from a point to the ground surface also constitutes a critical surface. This property is of crucial importance to the development of a numerical procedure for simulating the CSF.

It is found in earth pressure problems that the critical slip surface is unique, which means there are no two surfaces that yield exactly the same extremum lateral force or thrust force. This property leads to the fact that the critical slip surfaces in the CSF will not intersect each other. For example, line $\mathrm{BDC}_{3}$ as shown in Fig. 3(a) may be directly excluded from being considered a candidate for the critical surface. If it is (through D), then there would be two critical slip surfaces yielding extremum thrust force $P_{\mathrm{D}}$ (not shown in Fig. 3(a)) simultaneously. This would not happen in earth pressure problems involving homogeneous or nearly homogeneous soils.

In the method of slices, the slip surface as well as the critical surface is approximated by a series of straight-line segments across individual slices, say SB in Fig. 3(b). From the above conclusion, the inclination of the line segment SB, $\alpha_{\mathrm{S}}$, is also uniquely determined. $\alpha_{\mathrm{S}}$ is defined as the critical slip direction for point $\mathrm{S}$. Corresponding to any point in the CSF there are two quantities: the critical slip direction and the critical thrust force. It can be seen from equation (1) that the critical quantities of any point are dependent only on the critical thrust forces along the upper slice boundaries. As shown in Fig. 3 (b), if critical thrusts at all the points along the slice boundary through $\mathrm{B}$ have been determined in the last stage, then $\alpha_{\mathrm{S}}$ and $P_{\mathrm{S}}$ for point $\mathrm{S}$ can also be calculated by using equation (1), with $\alpha_{\mathrm{S}}$ selected such that $P_{\mathrm{S}}$ is a maximum or a minimum in the active or passive case.

The principle of extremum thrust force is derived directly from examining the recurrence relation between interslice forces. This principle is identical with the principle of optimality (Bellman, 1957), on the basis of which an effective optimisation technique (dynamic programming) was developed for solving types of sequential multistage optimisation problems. Dynamic programming was first adopted by Baker (1980) for locating the critical slip surface in slope stability analysis. The authors proposed the concept of the CSF in slopes by using the principle of optimality, and developed a special numerical technique (this technique will be described later in this paper) different from the conventional dynamic programming algorithm to simulate the CSF in slopes (Zhu, 1997; 1999). 


\section{NUMERICAL PROCEDURE FOR SIMULATING THE CSF}

It is known from the previous section that for any point in the CSF there exists a critical slip direction associated with a critical thrust force. Critical slip directions of a large number of points may sufficiently dictate the whole pattern of the CSF, and thus form the starting point for a numerical simulation procedure that attempts to assess the critical slip directions of a finite number of points uniformly distributed in the calculation region and trace the CSF through these discretised directions. To obtain a clear presentation in this section, the inclinations of thrust forces are assumed to be specified by a proper interslice function. The CSF obtained in this section is therefore not rigorous since the moment equilibrium condition (equation (6)) will not be taken into account. Problems of how to determine the thrust force inclinations and obtain a rigorous CSF will be discussed in detail later in separate sections of the paper, with regard to the situations under consideration.

\section{Discretisation of backfill}

Consider a soil-wall system as shown in Fig. 4, some geometrical parameters being defined in Fig. 1. As illustrated in Appendix 1, the backfill material at limiting state may be divided into two zones: the Rankine zone and the transition zone. The calculation region is delimited within a parallelogram of size $S_{x} \times S_{y}$ in the $x$ and $y$ directions respectively. According to the theory of plasticity, within the transition zone all possible failure surfaces would not pass beyond the utmost slip line, which can be described by a log-spiral function as indicated in Fig. 4. Values of $S_{x}$ and $S_{y}$ are estimated as follows:

$$
\begin{aligned}
S_{x} & \cong 1 \cdot 2 L \cdot \exp (-\psi \cdot \tan \bar{\phi}) \cdot \sin \psi \\
S_{y} & \cong 1 \cdot 2 \max \{L \cdot \exp (-\xi \cdot \tan \bar{\phi}) \cdot[\cos \xi+\sin \xi \cdot \tan (\beta-\omega)]\} \\
0 & \leqslant \xi \leqslant \psi
\end{aligned}
$$

The calculation region is then divided into $N-1$ slices with $N$ boundaries including the wall itself; along each slice bound- ary $M^{\prime}$ grid points are specified ( $M$ points on the wall). The slice width $b$ is equal to $S_{x} /(N-1)$, and the spacing of grid points in the $y$ direction $(d)$ is equal to $L /(M-1)$. In actual computation, only $M$ and the ratio $b / d$ are required to be prescribed a priori. According to the authors' experience, the ratio $b / d$ is suggested to be in the range $2-4$, while the selection of $M$ is dependent on the compromise between accuracy and cost of computation time. Generally, sufficient accuracy of numerical computation is ensured if $M$ is more than 40. Results of a large number of computations revealed that the accuracy would not decrease beyond practical tolerance if $M$ equals 20 or so. Even though $M$ is set to be 11 , as is the case in Fig. 4, the computation results are still of practical consequence.

\section{Determination of critical slip directions and thrust forces}

The critical slip directions as well as the critical thrust forces for grid points can be determined in a multi-stage manner by using the principle of extremum thrust force. For point $(i, j)$, the critical slip direction and critical thrust force are denoted as $\alpha^{\mathrm{c}}{ }_{i, j}$ and $P_{i, j}^{\mathrm{c}}$ (of components $P^{\mathrm{c}}{ }_{i, j}$ in the $x$ and $y$ directions: $\left.X^{\mathrm{c}}{ }_{i, j}, Y^{\mathrm{c}}{ }_{i, j}\right)$ respectively.

For grid points on the first boundary, the slip surfaces pointing upwards to the ground may be considered straight, so that the corresponding slip directions and critical thrusts can be directly determined by means similar to that in the Coulomb theory. The critical quantities for points on the second and onward slice boundaries can be calculated as follows, noting that $P^{\mathrm{c}}{ }_{1, j}=0$, and $\alpha^{\mathrm{c}}{ }_{1, j}$ may be set as $\alpha_{0}$, as determined by equation (18) in Appendix 1.

As a typical example, values of $P_{i, j}^{\mathrm{c}}$ and $\alpha^{\mathrm{c}}{ }_{i, j}$ are to be calculated assuming that the critical quantities for all points from $(1,1)$ to $(i-1, j)$ have been determined at previous stages as indicated in Fig. 5(a). As shown in Fig. 5(b), given a trial value of $\alpha^{\mathrm{c}}{ }_{i, j}$ as $\alpha_{i, j}$, the corresponding slice base from point $(i, j)$ intersects the $(j-1)$ th slice boundary at a distance equal to $r$-d from point $(k, j-1), r$ being positive when the intersection point is located below $(k, j-1)$ and vice versa. $k$ and $r$

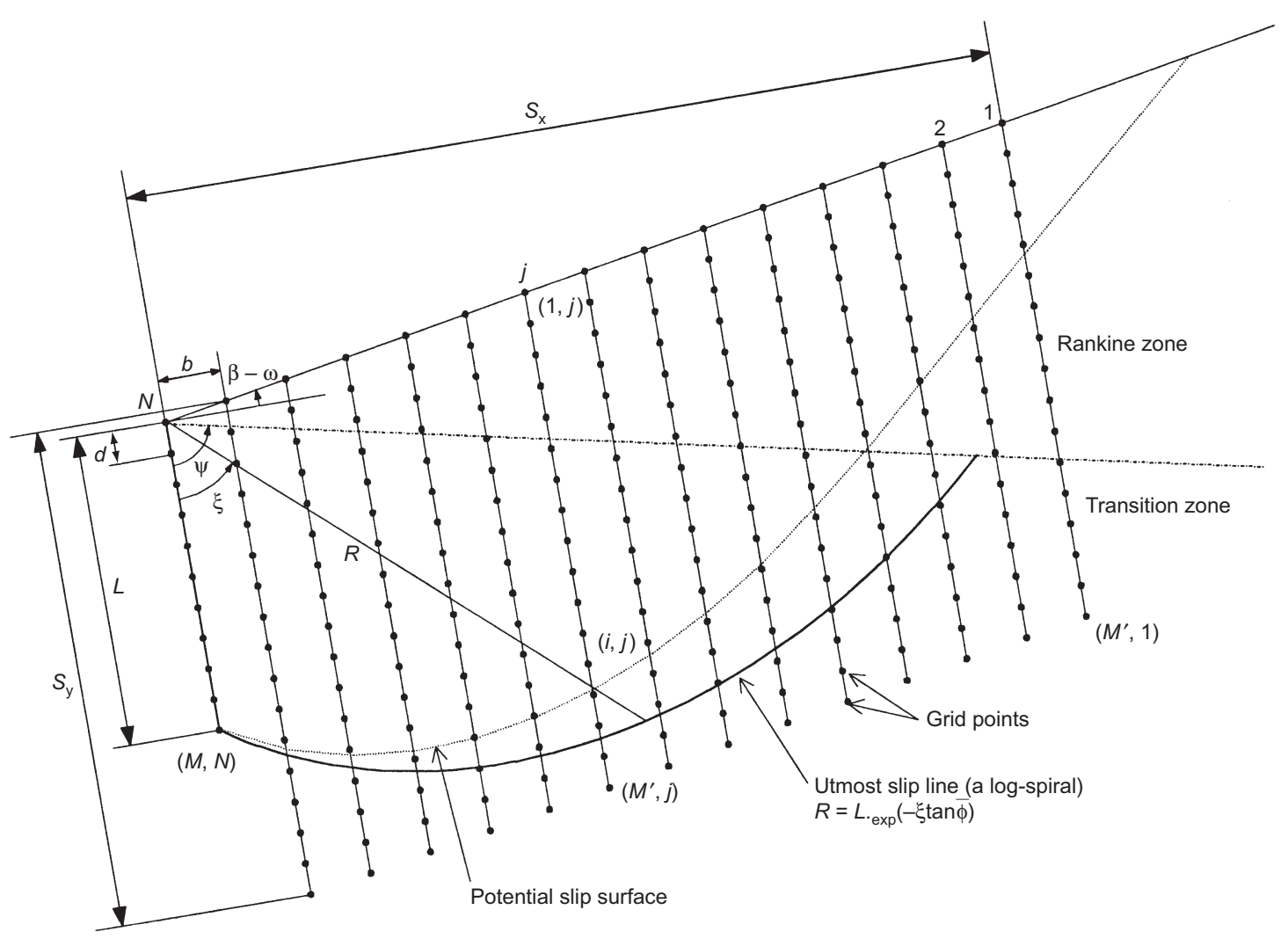

Fig. 4. Discretisation of the backfill 

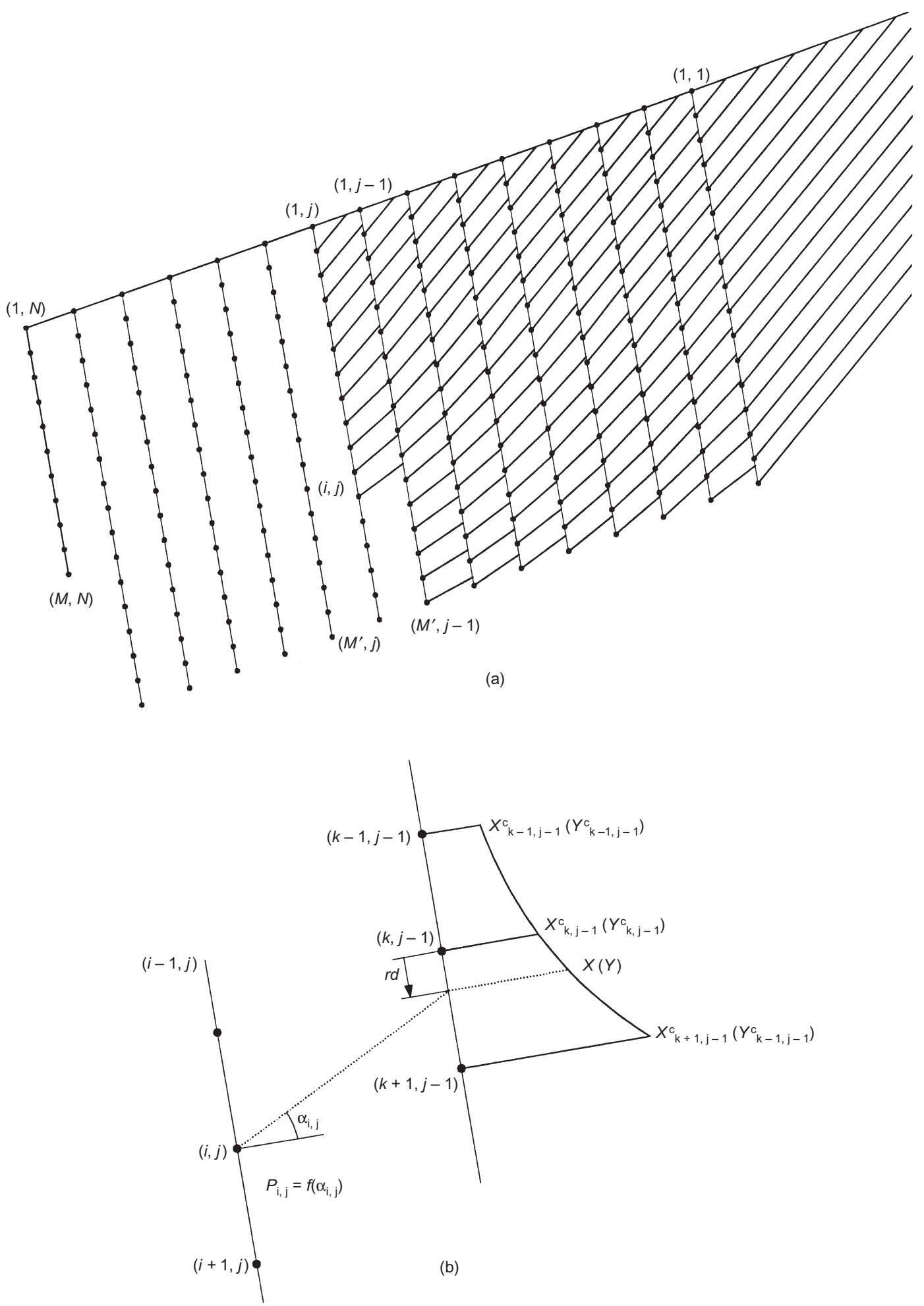

Fig. 5. Determination of critical slip directions and thrust forces: (a) critical slip directions partly completed; (b) interpolation of thrust forces

can be obtained by the following equations, which may be readily derived from geometrical relations:

$$
\begin{aligned}
k & =\operatorname{Max}\left\{2, i+\operatorname{Int}\left[\frac{b}{d} \tan (\beta-\omega)-\frac{b}{d} \tan \alpha_{i, j}\right]\right\} \\
r & =(i-k) d+b \tan (\beta-\omega)-b \tan \alpha_{i, j}
\end{aligned}
$$

The thrust force $P_{i, j}$ corresponding to $\alpha_{i, j}$ may be calculated using the recurrence relation between interslice forces (equation (2)). It is obvious that the determination of the thrust force (its components: $X, Y)$ on the $(j-1)$ th slice boundary is the key issue to the calculation of $P_{i, j} . X$ and $Y$ are computed using an interpolation function as follows:

$$
\begin{aligned}
X= & X_{k-1, j-1}^{\mathrm{c}} \cdot\left(-\frac{r}{2}+\frac{r^{2}}{2}\right) \\
& +X_{k, j-1}^{\mathrm{c}} \cdot\left(1-r^{2}\right)+X_{k+1, j-1}^{\mathrm{c}} \cdot\left(\frac{r}{2}+\frac{r^{2}}{2}\right) \\
Y= & Y_{k-1, j-1}^{\mathrm{c}} \cdot\left(-\frac{r}{2}+\frac{r^{2}}{2}\right) \\
& +Y_{k, j-1}^{\mathrm{c}} \cdot\left(1-r^{2}\right)+Y_{k+1, j-1}^{\mathrm{c}} \cdot\left(\frac{r}{2}+\frac{r^{2}}{2}\right)
\end{aligned}
$$

It is shown here that $P_{i, j}$ can be regarded as an implicit function 
of $\alpha_{i, j}$ : that is, $P_{i, j}=f\left(\alpha_{i, j}\right)$. The determination of $P^{\mathrm{c}}{ }_{i, j}$ is obtained by maximisation (or minimisation) of $P_{i, j}$ with respect to $\alpha_{i, j}$ in the active (or passive) case:

$$
P_{i, j}^{\mathrm{c}}=f\left(\alpha_{i, j}^{\mathrm{c}}\right)= \begin{cases}\operatorname{Max} f\left(\alpha_{i, j}\right) & \text { in the active case } \\ \operatorname{Min} f\left(\alpha_{i, j}\right) & \text { in the passive case }\end{cases}
$$

Such an optimisation can be realised by trial and error since it involves only a single variable. A good starting value of $\alpha_{i, j}$ is suggested as $\alpha^{\mathrm{c}}{ }_{i-1, j}$.

Repeat the above procedure until the critical slip directions and thrust forces of all grid points are obtained. All critical slip directions are stored for further use, while only the critical thrust forces of those points on two adjacent slice boundaries are required to be recorded and updated successively in the process. Finally, the thrust forces for points on the wall represent an approximation to local resultants of earth pressures summing from those points to the top of the wall.

\section{Tracing of CSF}

The critical slip directions of all grid points, as shown in Fig. 6(a), constitute a field of discretised pattern through which continuous critical slip surfaces (dotted lines in Fig. 6(a)) can be traced one by one. The tracing procedure is as follows:

Commencing from a grid point, say A (Fig. 6) on the wall (i.e. the $N$ th slice boundary), a critical slip surface is traced to the $(N-1)$ th slice boundary at $\mathrm{B}$, which is located between two adjacent grid points $B_{1}$ and $B_{2}$. This is further traced to $C$, and then $\mathrm{D}$, and so on. The positions of $\mathrm{C}$ and $\mathrm{D}$ are determined by linear interpolation as indicated in Fig. 6(b) using the following relation:

$$
\frac{\overline{\mathrm{B}_{1} \mathrm{~B}}}{\overline{\mathrm{BB}_{2}}}=\frac{\overline{\mathrm{B}_{3} \mathrm{C}}}{\overline{\mathrm{CB}_{4}}}, \quad \frac{\overline{\mathrm{C}_{1} \mathrm{C}}}{\overline{\mathrm{CC}_{2}}}=\frac{\overline{\mathrm{C}_{3} \mathrm{D}}}{\overline{\mathrm{DC}_{4}}}
$$

where $\mathrm{B}_{3}, \mathrm{~B}_{4}, \mathrm{C}_{3}$ and $\mathrm{C}_{4}$ are determined immediately from the critical slip directions of $B_{1}, B_{2}, C_{1}$ and $C_{2}$ respectively. The
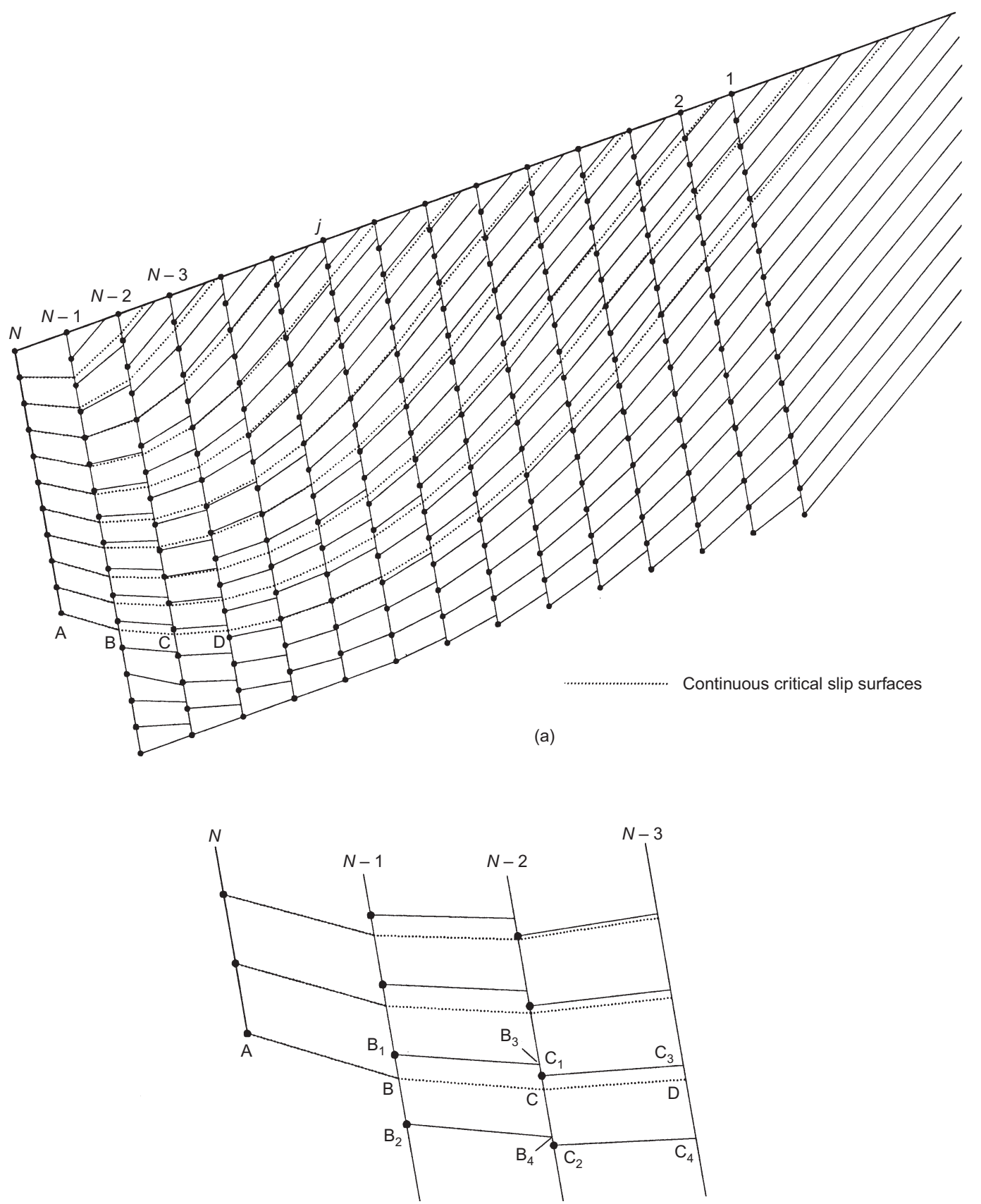

(a)

Fig. 6. Tracing of CSF: (a) CSF and critical slip directions; (b) details of tracing 
tracing is terminated at the ground surface, thus resulting in a continuous critical slip surface. By repeating the tracing process for the rest of the grid points on the wall, the CSF in the sense of force equilibrium is thus obtained. The CSF in a rigorous sense will be discussed next, but the procedure presented in this section constitutes a major part of the numerical simulation of the CSF.

\section{CSF IN THE CASE OF WEIGHTLESS SOIL}

This special case has been dealt with by the classical theory of plasticity, and closed-form solutions are widely available (Sokolovski, 1965; Powrie, 1997). The purpose of this section is to validate the method of CSF by comparing numerical solutions with the theoretical ones.

In this case, the distribution of earth pressures along the wall is uniform, so that the resultant lateral force acts at the midpoint of the wall. All critical slip surfaces are expected to be of a common shape: thus a common interslice force function involving a common value of $m$ is able to describe the inclinations for all surfaces of the CSF. The calculation of the CSF is performed step by step as follows:

(a) Discretise the backfill. This has been illustrated in the previous section.

(b) Choose an interslice force function as suggested in Appendix 1.

(c) Assume a starting value of $m$ for the interslice force function. Unity of $m$ is suggested if lacking experience. (d) Calculate the CSF without consideration of the moment equilibrium condition using the numerical procedure in the preceding section.

(e) Take the critical slip surface through the wall toe obtained in step $(d)$ as a specified surface and re-divide the sliding mass above it into a larger number of slices to calculate the moment with sufficient accuracy. Calculate next the lateral force $P$ and parameter $m$ by using the rigorous method of slices previously presented in the paper. The constants in the moment equilibrium condition (equation 4) become $C_{1}=-\frac{1}{2} L \cos \delta, C_{2}=0$.

$(f)$ With the modified value of $m$ in step (e), repeat steps $(d)$ and $(e)$ until the difference in the value of $P$ between two successive iterations is within a specified tolerance. It is found that both $P$ and $m$ converge to stable values rapidly after three to five iterations. The CSF at the end of this step is then accepted.

(g) Using the finite difference method, compute the numerical distribution of earth pressures from the critical thrust forces of grid points on the wall.

\section{Examples}

Example 1. The geometrical and strength parameters are presented in Fig. 7. The values of lateral forces by using the theory of plasticity are: $P_{\mathrm{a}}=315 \mathrm{kN} / \mathrm{m}, \quad P_{\mathrm{p}}=5804 \mathrm{kN} / \mathrm{m}$. Taking $M=81$ and $b / d=4$, the ACSF and PCSF (solid lines) in Fig. 7(a) and (b) respectively show that they are nearly coincident with the theoretical slip lines (dotted lines). The

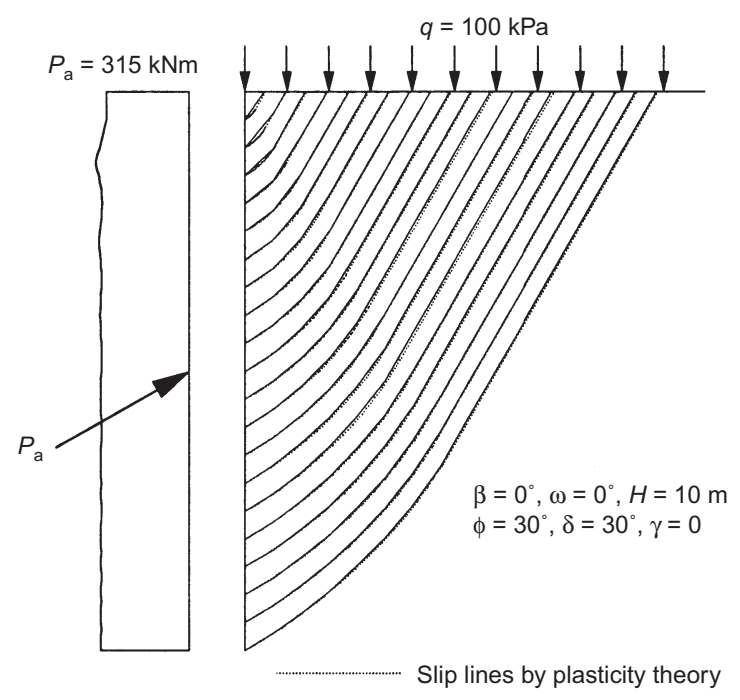

(a)

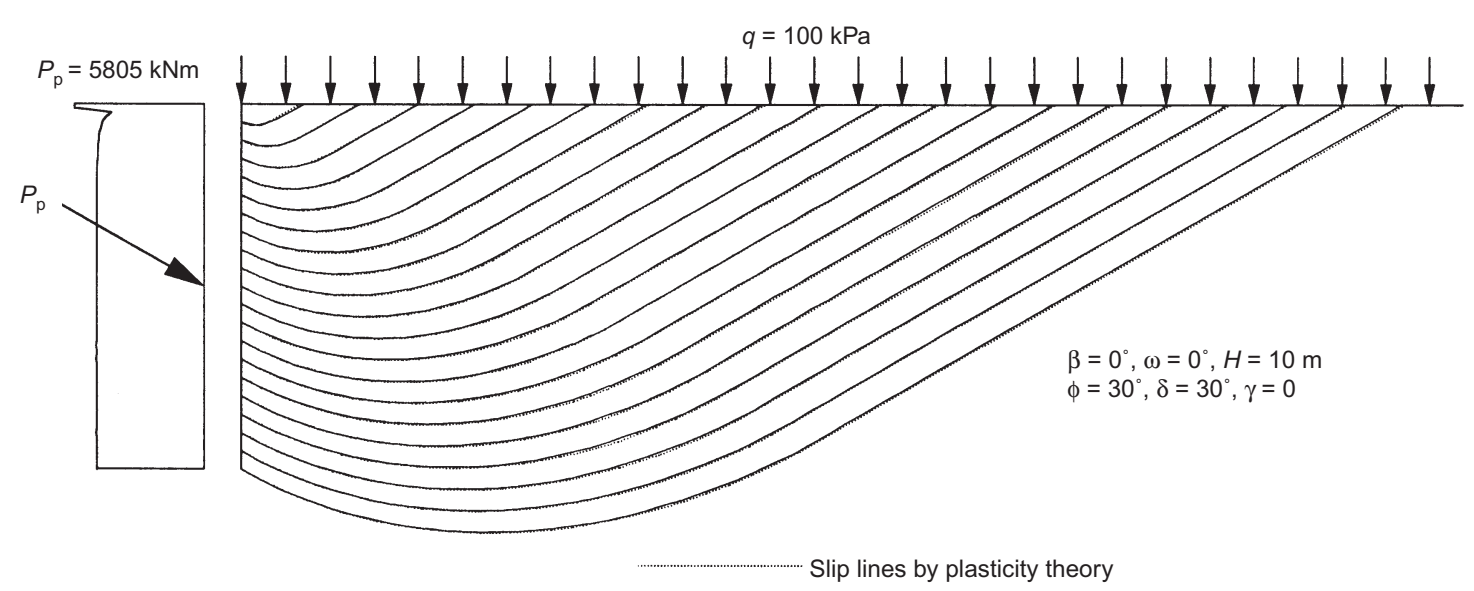

(b)

Fig. 7. CSF in Example 1: (a) ACSF; (b) PCSF 
computed lateral forces are $P_{\mathrm{a}}=315 \mathrm{kN} / \mathrm{m}, P_{\mathrm{p}}=5805 \mathrm{kN} / \mathrm{m}$, which are identical with the theoretical solutions. The numerical distributions of earth pressures are approximately uniform except when they are near the top of the wall. This is due to the inadequate number of slices relative to the limited local height of the wall, but it has no effect on the total lateral forces.

Example 2. This is a typical example often encountered in the calculation of the bearing capacity of strip footings. The PCSF shown in Fig. 8 is in good agreement with the theoretical slip lines, and the calculated value of $P_{\mathrm{p}}$ is equal to $10628 \mathrm{kN} / \mathrm{m}$ as compared with $10624 \mathrm{kN} / \mathrm{m}$ by the theory of plasticity.

\section{CSF IN THE CASE OF ZERO SURCHARGE}

When there is no surcharge acting upon the surface of a cohesionless backfill, the distribution of earth pressures along the wall is hydrostatic and the point of application of the resultant lateral force lies at the lower-third point of the wall. The shape of the failure surface should be independent of the height of the wall. This therefore leads to the use of a common interslice force function with a common value of parameter $m$ for the calculation of the CSF. The procedure for simulating the CSF presented in the section above is also applicable to this case, noting that the moment equilibrium condition used in step (e) changes to $C_{1}=-\frac{1}{3} L \cos \delta, C_{2}=0$. By setting the unit weight $\gamma$ and the wall height $H$ as arbitrary values, and then calculating the lateral force $P_{\mathrm{a}}\left(P_{\mathrm{p}}\right)$ for the entire wall, the earth pressure coefficient $K_{\mathrm{a}}\left(K_{\mathrm{p}}\right)$ is obtained and is associated with a set of strength and geometrical parameters: $\phi, \delta, \beta$ and $\omega$. Herein $K_{\mathrm{a}}\left(K_{\mathrm{p}}\right)$ is defined as $P_{\mathrm{a}} / 0.5 \gamma H^{2}\left(P_{\mathrm{p}} / 0 \cdot 5 \gamma H^{2}\right)$, and those coefficients given by Caquot \& Kerisel (1948) and obtained by using the Coulomb theory are denoted as $K_{\mathrm{a}}{ }^{\mathrm{C}-\mathrm{K}}$ $\left(K_{\mathrm{p}}^{\mathrm{C}-\mathrm{K}}\right)$ and $K_{\mathrm{a}}^{\mathrm{C}}\left(K_{\mathrm{p}}^{\mathrm{C}}\right)$ respectively.

\section{Examples}

Two examples (Examples 3, 4) are presented in this case, with the results shown in Figs 9 and 10, respectively. The number of grid points on the wall, $M$, is taken as 21 , and the ratio $b / d$ is set at 2 in calculating the CSF. It is shown that both active and passive earth pressure coefficients by the method of CSF differ slightly from those given by Caqout \& Kerisel, while the Coulomb theory either overestimates (Example 3) or fails to calculate the passive earth pressure coefficient (Example 4). The numerical distribution of earth pressure is exactly hydrostatic as predicted by the theory of plasticity.

\section{CSF IN THE CASE OF SURCHARGE}

The distribution of earth pressures is no longer linear in the case of backfill carrying surcharge on its surface. It is impos- sible in this case to use a single interslice force function with a common value of parameter $m$ to describe the variation of inclination of interslice force throughout the whole sliding mass since no geometrical similitude exists among the critical slip surfaces of the CSF. However, the CSF can be obtained by determining each of the critical slip surfaces successively using the same simulation procedure illustrated in the previous sections with the moment equilibrium condition altered in consistence with the distribution of earth pressures.

Referring to Fig. 11, the distribution of earth pressures along the wall is approximated by a series of linear segments with pressure $p_{1}, p_{2}, \ldots, p_{\mathrm{MD}}$ at joints $1,2, \ldots, \mathrm{MD}$. The spacing of joints is $\Delta L=L /(\mathrm{MD}-1)$. To each joint, say $j$, there corresponds a critical slip surface that produces a lateral force $P_{j}$ acting at a distance $r_{j}$ from the joint. It is obvious that

$$
\begin{aligned}
& P_{j+1}-P_{j}=\frac{p_{j+1}+p_{j}}{2} \cdot \Delta L \\
& P_{j+1} \cdot r_{j+1}=P_{j}\left(r_{j}+\Delta L\right)+\frac{1}{2} p_{j} \cdot \Delta L^{2}+\frac{1}{6}\left(p_{j+1}-p_{j}\right) \cdot \Delta L^{2}
\end{aligned}
$$

Combining the above two equations results in

$$
\begin{aligned}
& P_{j+1} \cdot r_{j+1}=\frac{\Delta L}{3} P_{j+1}+P_{j}\left(r_{j}+\frac{2}{3} \Delta L\right)+\frac{1}{6} p_{j} \cdot \Delta L^{2} \\
& p_{j+1}=\frac{2}{\Delta L}\left(P_{j+1}-P_{j}\right)-p_{j} \\
& r_{j+1}=\frac{\Delta L}{3}+\frac{P_{j}}{P_{j+1}}\left(r_{j}+\frac{2}{3} \Delta L\right)+\frac{p_{j}}{6 P_{j+1}} \cdot \Delta L^{2}
\end{aligned}
$$

The pressure $p_{1}$ at the top of the wall is identical to the density of earth pressure in the case of weightless soil with surcharge $q$. The value of $p_{1}$ can be obtained by means of the numerical procedure in the preceding, or directly by using the theory of plasticity.

Assuming that the critical slip surfaces through joints 2 to $j$ have been determined in the previous stages, with values of $P_{j}$, $p_{j}$ and $r_{j}$ obtained, now calculate the critical slip surface through joint $j+1$ and its lateral force $P_{j+1}$. Such a surface can be regarded as the outermost critical slip surface of the CSF for a wall of oblique height $j \Delta$. The process of calculation of this intermediate CSF is the same as before, except that the constants in the moment equilibrium condition (equation (4)) are as follows (referring to equations (3), (4) and (13)):

$$
\begin{aligned}
& C_{1}=-\frac{\Delta L}{3} \cdot \cos \delta \\
& C_{2}=-P_{j}\left(r_{j}+\frac{2}{3} \Delta L\right) \cdot \cos \delta-\frac{1}{6} p_{j} \cdot \Delta L^{2} \cdot \cos \delta
\end{aligned}
$$

With the obtained value of $P_{j+1}$, the values of $p_{j+1}$ and $r_{j+1}$ can be readily calculated from equations (14) and (15), respectively. This process is repeated until the critical slip surfaces for all joints as well as the corresponding earth pressures are

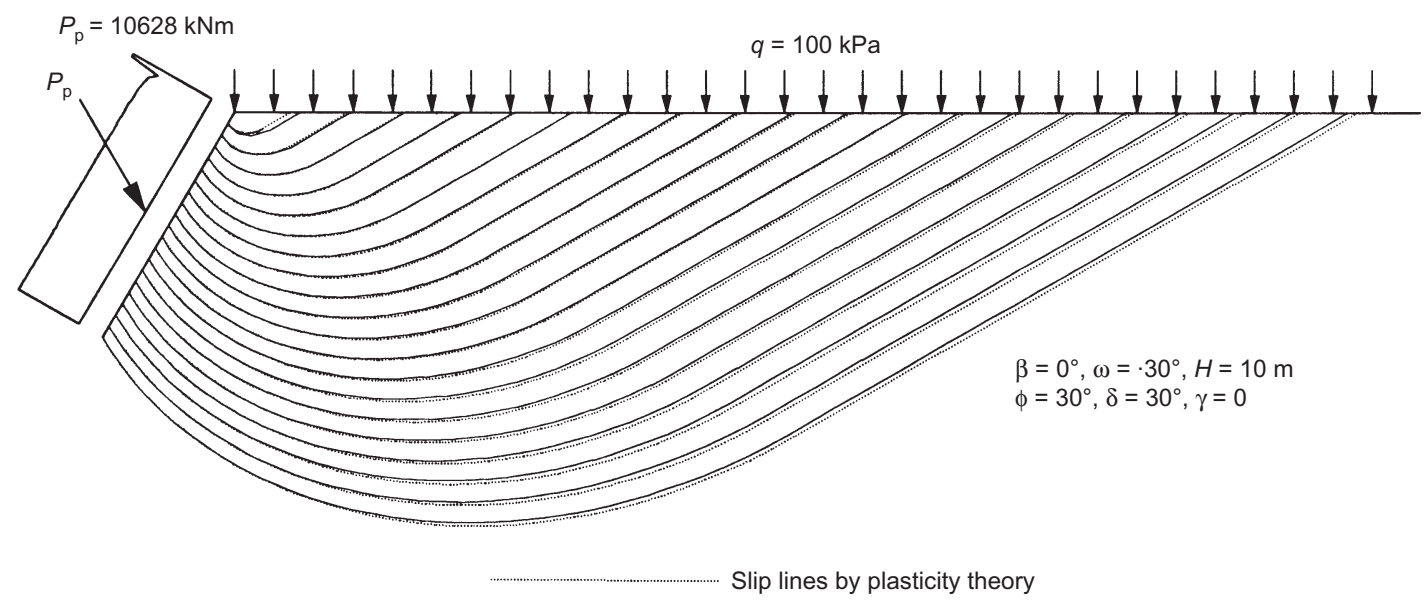

Fig. 8. PCSF in Example 2 

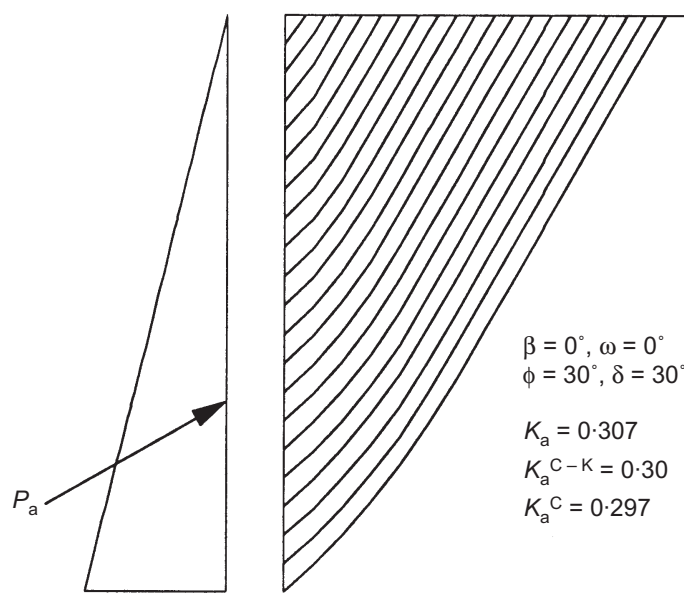

(a)
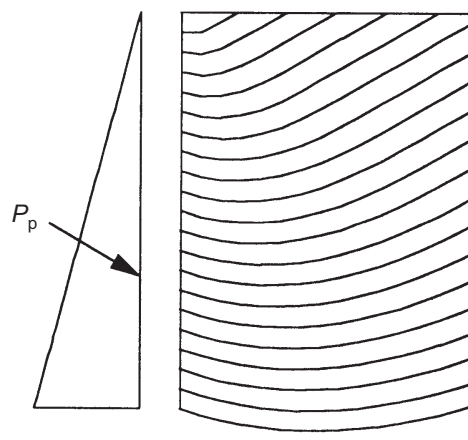

Fig. 9. CSF in Example 3: (a) ACSF; (b) PCSF $\phi=30^{\circ}, \delta=30$

$K_{\mathrm{a}}=0.307$

$\mathrm{K}_{\mathrm{a}} \mathrm{C}-\mathrm{K}=0.30$

$K_{\mathrm{a}} \mathrm{C}=0.297$ 

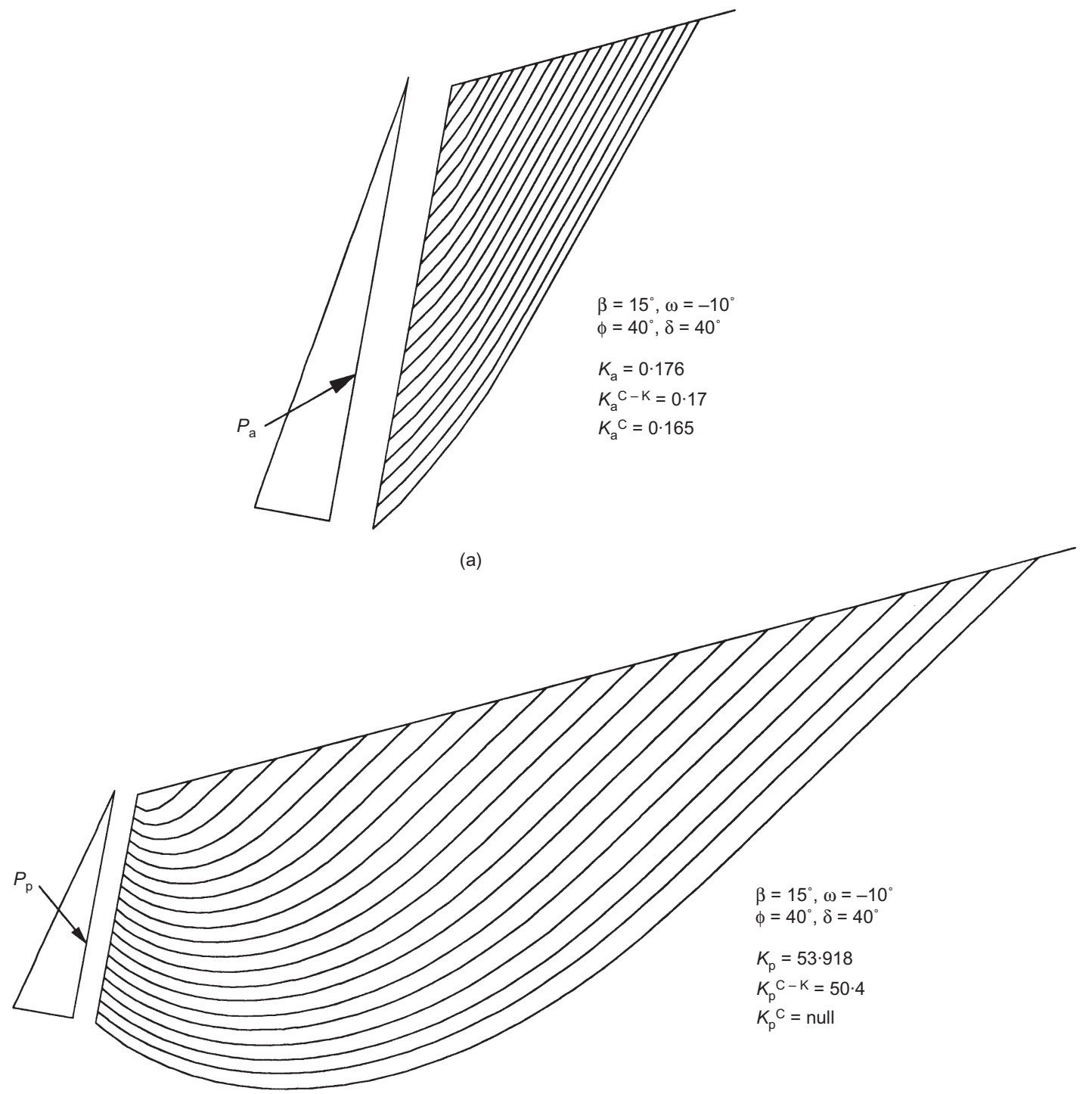

(b)

Fig. 10. CSF in Example 4: (a) ACSF; (b) PCSF

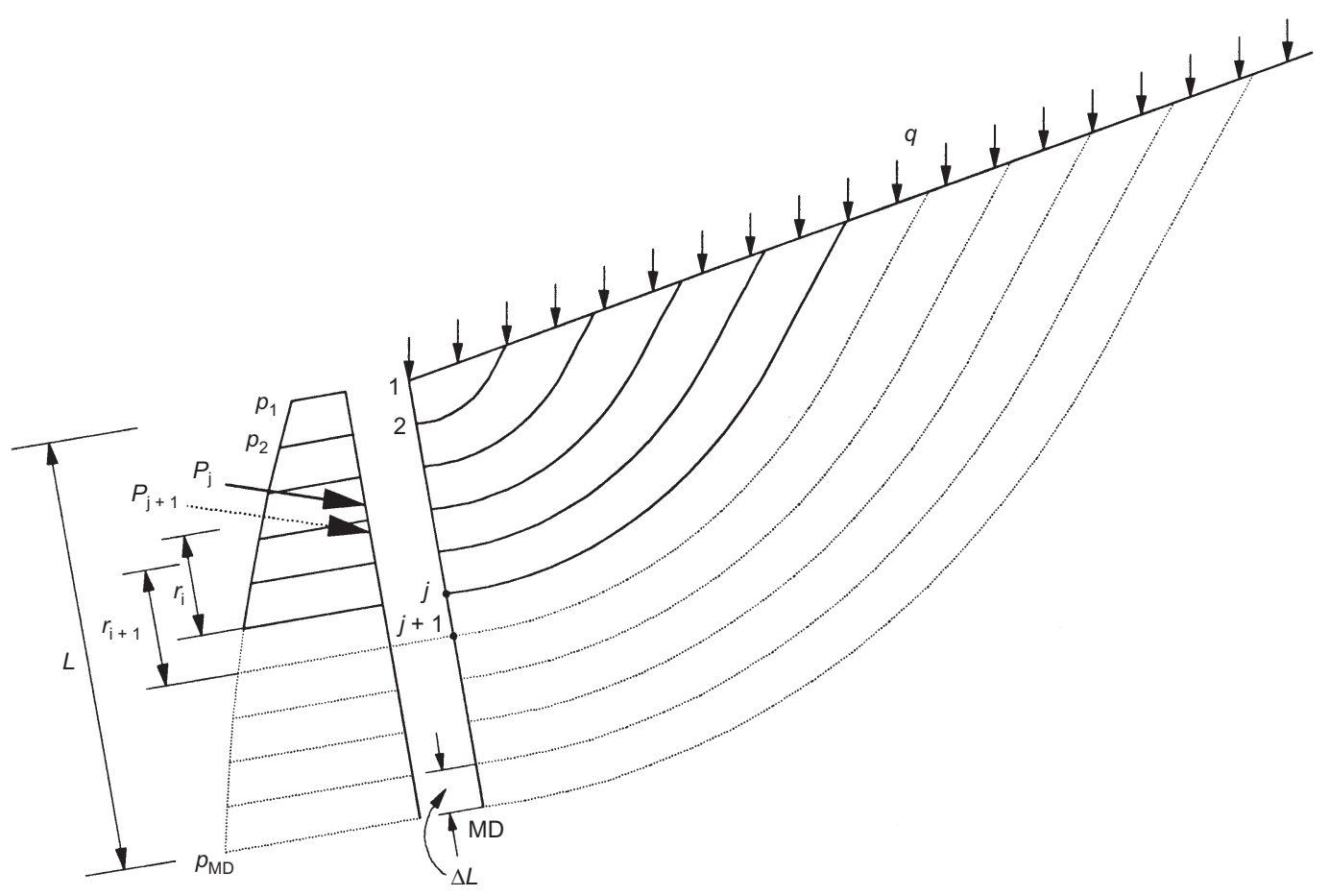

Fig. 11. Determination of CSF in the case of surcharge 


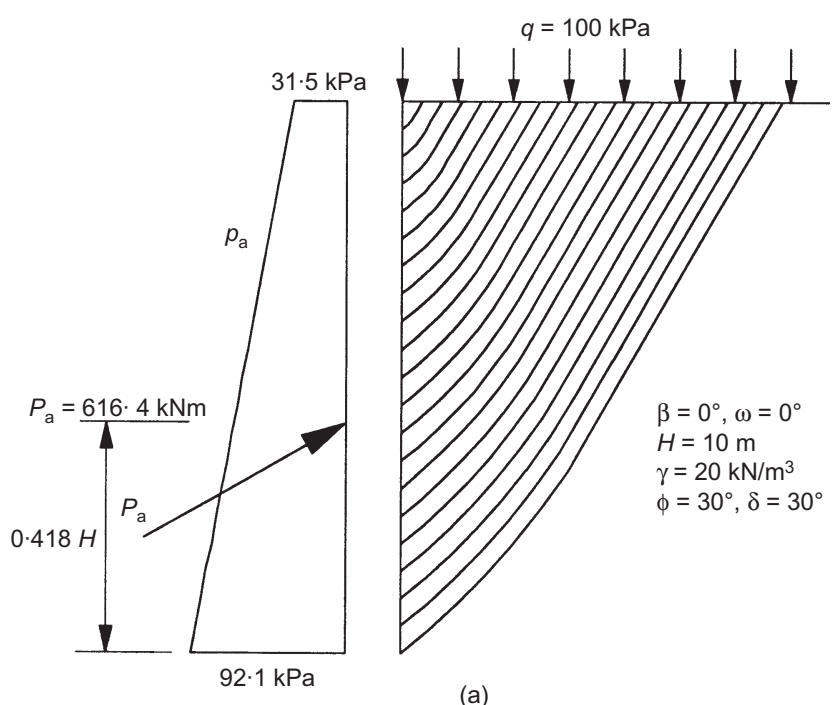

(a)

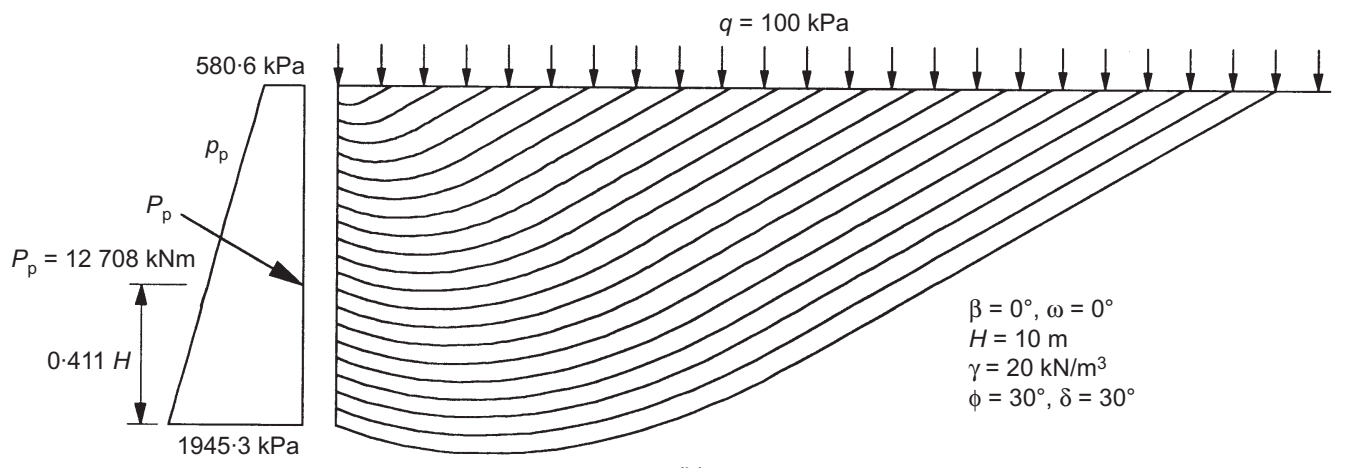

(b)

Fig. 12. CSF in Example 5: (a) ACSF; (b) PCSF

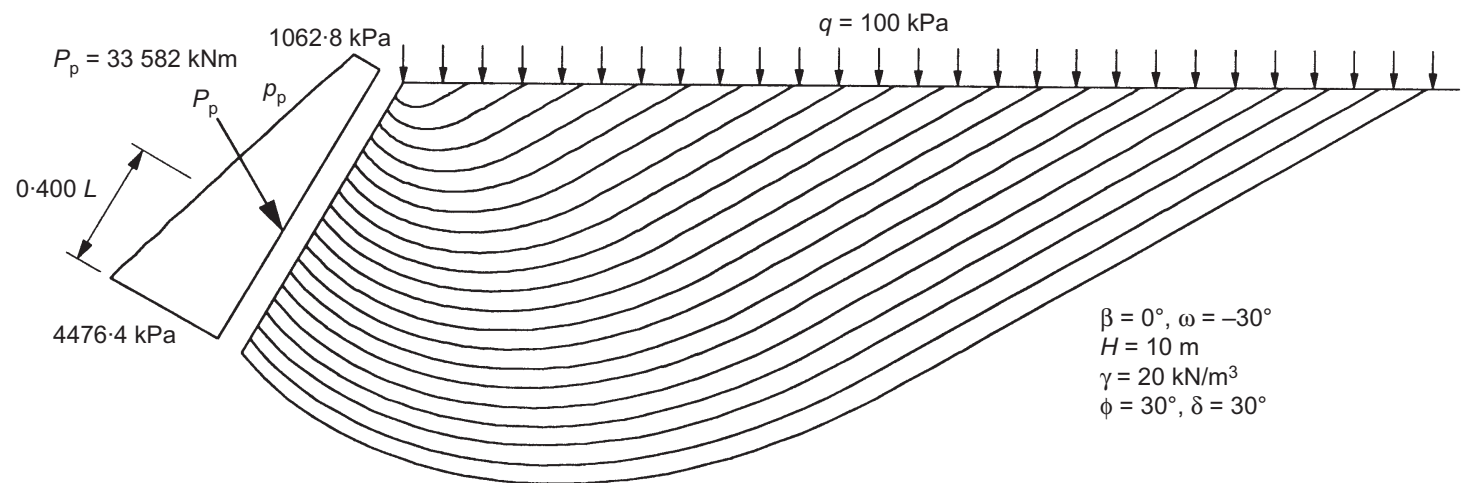

Fig. 13. PCSF in Example 6
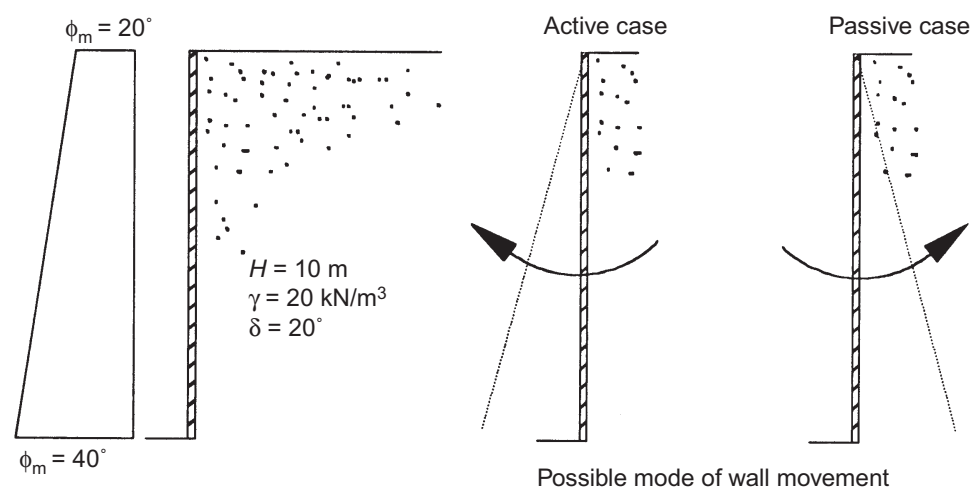

Fig. 14. Example 7: case of varying strength mobilisation 


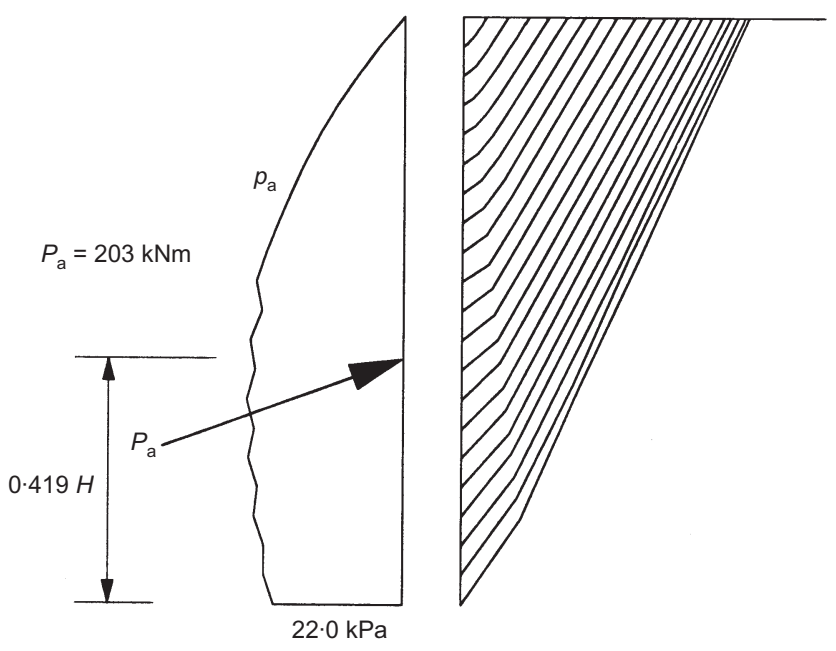

(a)

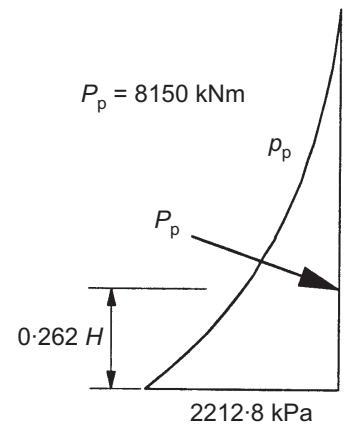

Fig. 15. CSF in Example 7: (a) ACSF; (b) PCSF vertical surcharge). It is found that the CSF can be in very close agreement with the closed-form slip lines by the theory of plasticity, and the numerical values of earth pressures are nearly identical with the theoretical solutions in spite of their essentially different theoretical backgrounds. It is justified to anticipate that the CSF method can still give highly accurate solutions to earth pressure problems widely encountered in practice where theoretical solutions are not available.

Furthermore, the CSF method shows promise in accommodating non-homogeneous backfill materials possessing both friction angle and cohesion under more complicated loading conditions.

\section{ACKNOWLEDGEMENTS}

The writers wish to thank Professor Q. Fang, Nanjing Engineering Institute, and Professor W. L. Liu, North Jiarong University and Professor Z. M. Zhang, Hohai University for their great help in the preparation and revision of this paper.

\section{APPENDIX 1. CHOICE OF INTERSLICE FORCE FUNCTION}

The potential sliding mass is divided into two zones, the Rankine zone and the transition zone, with a transition ray inclined at an angle $\psi$ to the wall, as shown in Fig. 16. Within the Rankine zone, the failure surface should be a straight line with a slope of $\alpha_{0}$ to the $x$ direction, and the

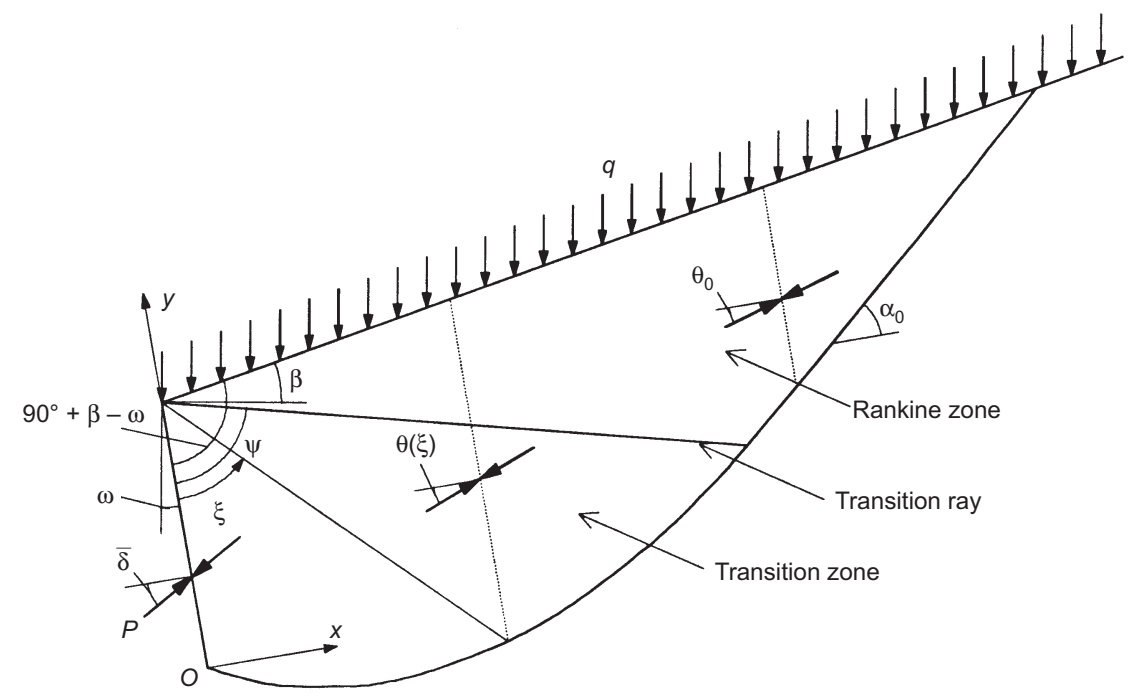

Fig. 16. Zone division for the sliding mass 
inclinations of thrust forces are a constant: that is, $\theta_{0}$. Within the transition zone, the failure surface is of a curved shape and the thrust force inclinations, $\theta(\xi)$, are varied from $\theta_{0}$ to $\delta$. The values of $\psi, \theta_{0}$ and $\alpha_{0}$ are determined in the following.

Consider an infinitesimal region near the top of the wall in which the influence of soil self-weight can be regarded as negligible. According to the theory of plasticity (Powrie, 1997), two distinct zones are developed in this region: zone 1 near the backfill surface, and zone 2 near the wall, as shown in Fig. 17(a), assuming that stress discontinuity does not occur Now investigate the state of stress within zone 1, which lies between two conjugate slip lines, $A_{1}$ and $A_{2}$, and the ground surface $A_{0}$, the tractions of which are indicated in the figure. It is well known that the traction on any plane parallel to the ground surface at an infinitesimal depth is inclined at an angle $\beta$ to its normal in the case of zero surcharge or vertical uniform surcharge (Terzaghi, 1943).

The state of stress within zone 1 can be represented by a Mohr diagram, as shown in Fig. 17(b). For simplicity, only the active case is considered in the Mohr diagram, while the derivations of relevant equations are applicable to both cases if $\bar{\phi}$ is replaced by $\bar{\phi}$. Thus $\phi$ is used in the following for general cases. The normal and tangential stresses on planes $A_{0}, A_{1}, A_{2}, A_{3}$ (parallel to the wall) in Fig. 17(a) correspond to points $A_{0}, A_{1}, A_{2}, A_{3}$ in the Mohr diagram in Fig. 17(b), the pole being at point P. From the geometrical relations in Fig. 17(b), we have

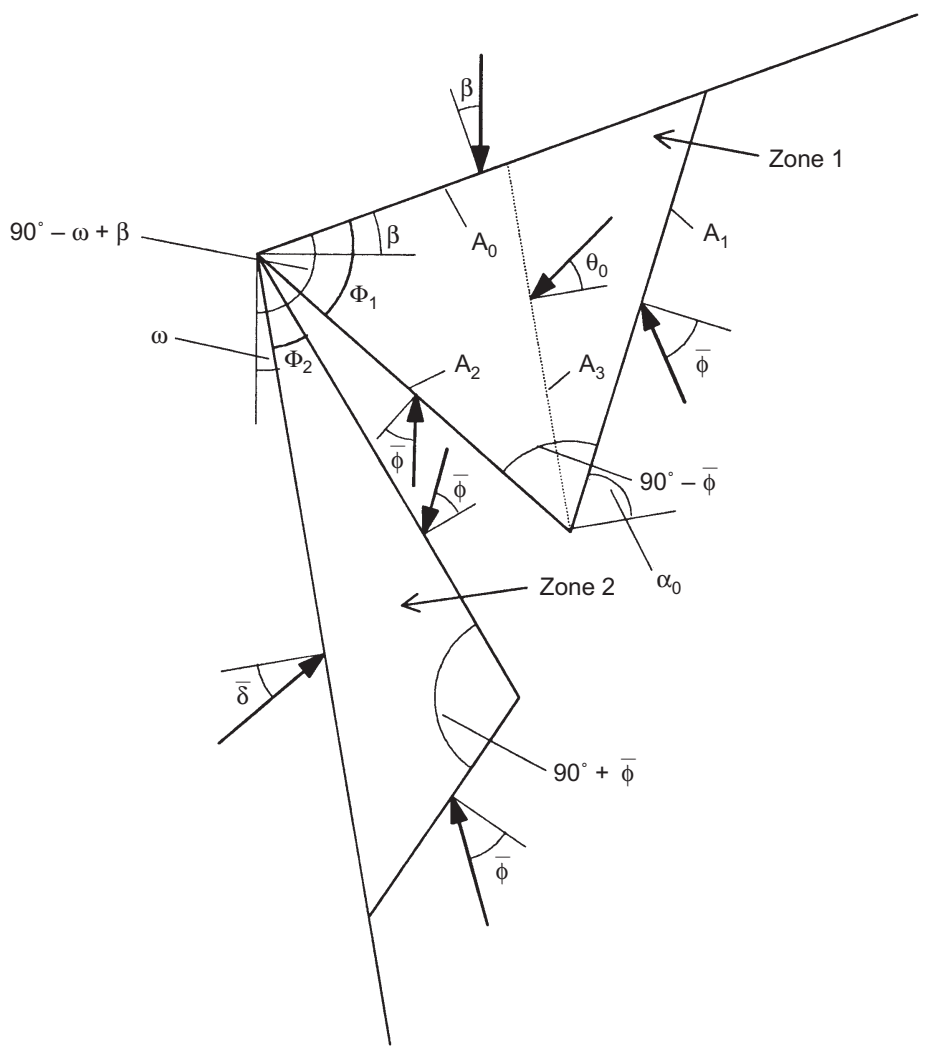

(a)

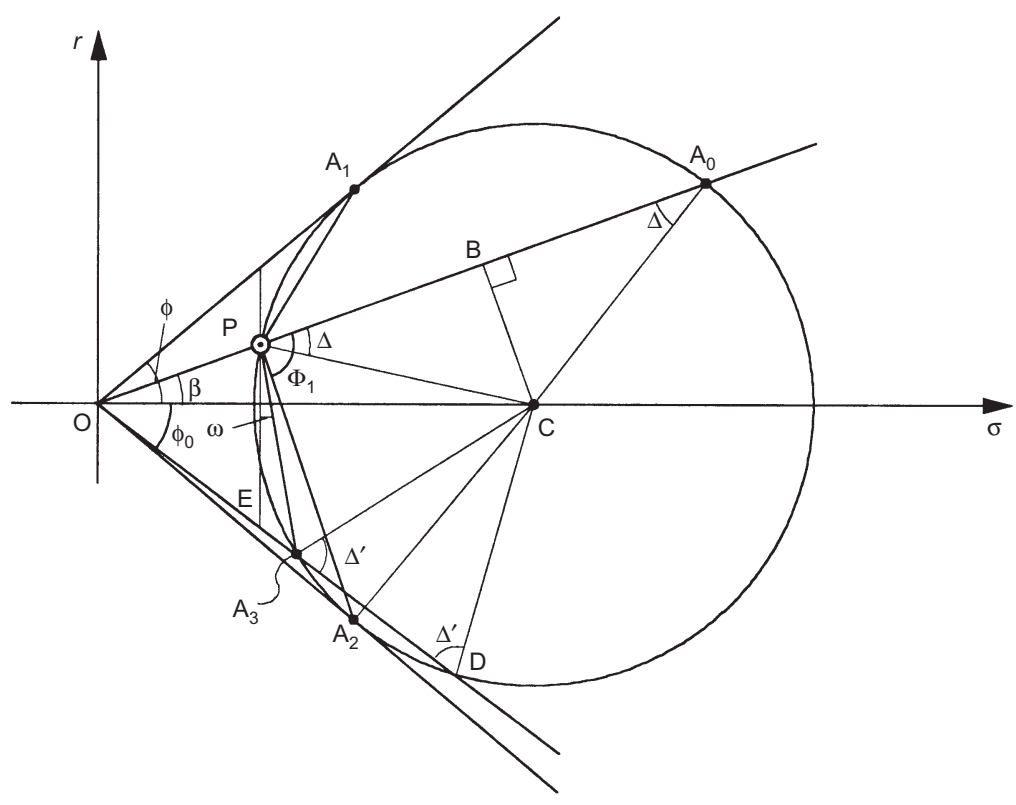

(b)

Fig. 17. Determination of the geometry of division zones: (a) case without stress discontinuity; (b) Mohr diagram for the state of stress in zone 1 (active case); (c) case of stress discontinuity: (d) Mohr diagram for locating stress discontinuity line (active case) 


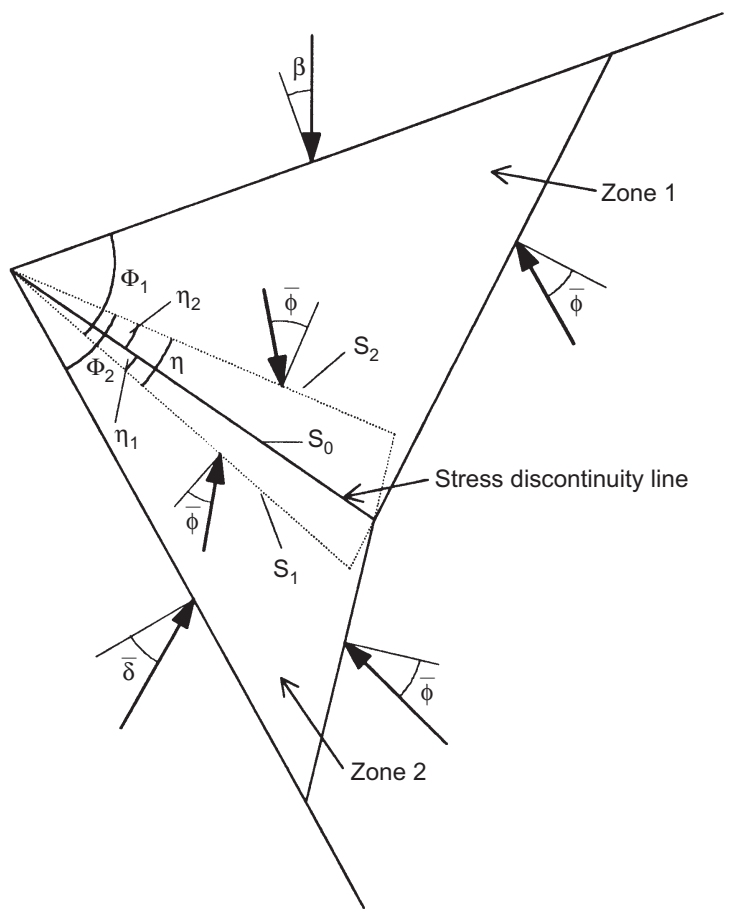

(c)

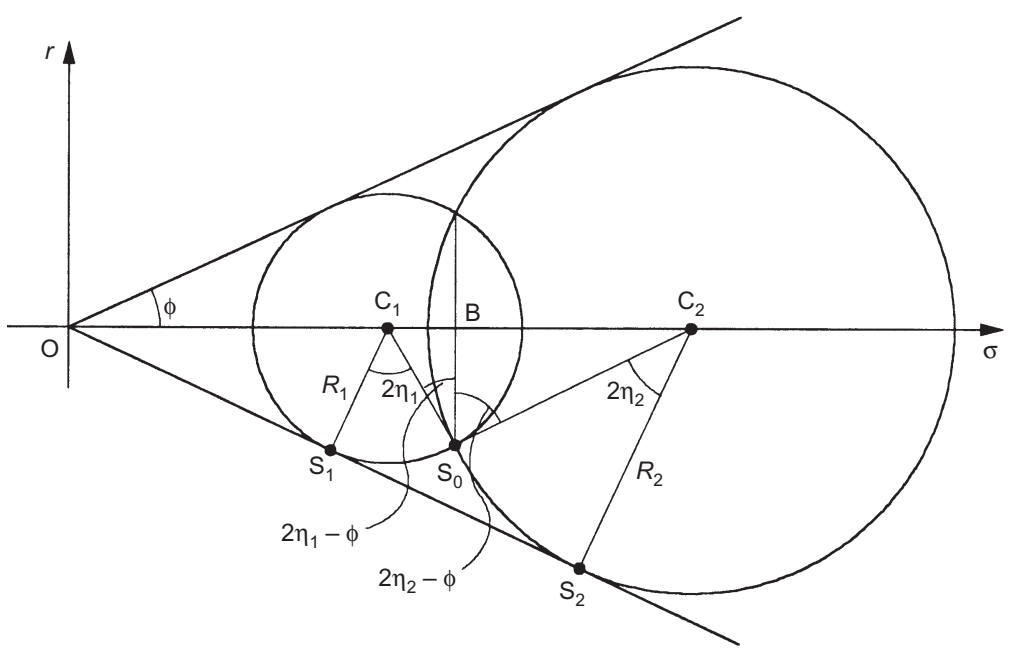

(d)

Fig. 17. (continued)

$$
\begin{aligned}
& \sin \Delta=\frac{\overline{\mathrm{BC}}}{\overline{\mathrm{CP}}}=\frac{\overline{\mathrm{OC}} \sin \beta}{\overline{\mathrm{OC}} \sin \bar{\phi}}=\frac{\sin \beta}{\sin \bar{\phi}}, \Delta=\arcsin \left(\frac{\sin \beta}{\sin \bar{\phi}}\right) \\
& \angle \mathrm{PCA}_{2}=\angle \mathrm{OCA}_{2}+\angle \mathrm{PCO}=90^{\circ}-\bar{\phi}+\Delta-\beta \\
& \angle \mathrm{PCA}_{2}+2 \cdot \angle \mathrm{CPA}_{2}=\angle \mathrm{PCA}_{2}+2\left(\Phi_{1}-\Delta\right)=180^{\circ}
\end{aligned}
$$

Thus the rake angle of zone $1, \Phi_{1}$, is expressed as

$$
\Phi_{1}=45^{\circ}+\frac{\bar{\phi}}{2}+\frac{1}{2} \arcsin \left(\frac{\sin \beta}{\sin \bar{\phi}}\right)+\frac{\beta}{2}
$$

It is obvious that

$$
\alpha_{0}=90^{\circ}+\beta-\Phi_{1}+\bar{\phi}
$$

Substituting $\Phi_{1}$ from equation (17) leads to

$$
\alpha_{0}=45^{\circ}+\frac{\bar{\phi}}{2}-\frac{1}{2} \arcsin \left(\frac{\sin \beta}{\sin \bar{\phi}}\right)+\frac{\beta}{2}
$$

By referring to Fig. 17(b) it can be seen that

$$
\angle \mathrm{PA}_{3} \mathrm{O}=\angle \mathrm{OEP}-\omega=90^{\circ}-\theta_{0}-\omega
$$

$$
\begin{aligned}
& \angle \mathrm{PA}_{3} \mathrm{O}+\Delta^{\prime}+\angle \mathrm{CPA}_{3}=180^{\circ} \\
& \angle \mathrm{CPA}_{3}=180^{\circ}-\Delta-\left(90^{\circ}-\beta\right)-\omega=90^{\circ}-\Delta+\beta-\omega
\end{aligned}
$$

These equations result in

$$
\Delta^{\prime}=\theta_{0}+\Delta-\beta+2 \omega
$$

Similar to $\Delta, \Delta^{\prime}=\arcsin \left(\sin \theta_{0} / \sin \bar{\phi}\right)$, and substituting into equation (19) and solving for $\theta_{0}$ yields

$$
\theta_{0}=\arctan \left\{\frac{\sin \left[\arcsin \left(\frac{\sin \beta}{\sin \bar{\phi}}\right)-\beta+2 \omega\right]}{\frac{1}{\sin \bar{\phi}}-\cos \left[\arcsin \left(\frac{\sin \beta}{\sin \bar{\phi}}\right)-\beta+2 \omega\right]}\right\}
$$

Similar to $\Phi_{1}, \Phi_{2}$ can be obtained by manipulating the Mohr diagram for zone 2, as follows:

$$
\Phi_{2}=45^{\circ}-\frac{\bar{\phi}}{2}-\frac{1}{2} \arcsin \left(\frac{\sin \bar{\delta}}{\sin \bar{\phi}}\right)+\frac{\bar{\delta}}{2}
$$

In the case of $\Phi_{1}+\Phi_{2}>90^{\circ}+\beta-\omega$, zones 1 and 2 run into each other, resulting in a stress discontinuity line, that is, $S_{0}$ in Fig. 17(c). The 
determination of geometrical parameters $\eta_{1}$ and $\eta_{2}$ defining the position of $S_{0}$ refers to the Mohr diagram in Fig. 17(d) (also corresponding to the active case for simplicity). The circle with centre $C_{1}$ represents the stress state for zone 1, while $C_{2}$ is for zone 2. According to geometrical relations:

$$
\begin{aligned}
& \overline{\mathrm{C}_{1} \mathrm{C}_{2}}=\frac{R_{2}-R_{1}}{\sin \bar{\phi}} \\
& \overline{\mathrm{C}_{1} \mathrm{C}_{2}}=\overline{\mathrm{C}_{1} \mathrm{~B}}+\overline{\mathrm{C}_{2} \mathrm{~B}}=R_{1} \sin \left(2 \eta_{1}-\bar{\phi}\right)+\mathrm{R}_{2} \sin \left(2 \eta_{2}+\bar{\phi}\right) \\
& \overline{\mathrm{S}_{0} \mathrm{~B}}=R_{1} \cos \left(2 \eta_{1}-\bar{\phi}\right)=\mathrm{R}_{2} \cos \left(2 \eta_{2}+\bar{\phi}\right) \\
& \eta=\eta_{1}+\eta_{2}
\end{aligned}
$$

$\eta_{1}$ and $\eta_{2}$ are obtained as

$$
\begin{aligned}
& \eta_{1}=\frac{\eta}{2}+\bar{\phi}-\frac{1}{2} \arcsin (\sin \bar{\phi} \cos \eta) \\
& \eta_{2}=\frac{\eta}{2}-\bar{\phi}+\frac{1}{2} \arcsin (\sin \bar{\phi} \cos \eta)
\end{aligned}
$$

where

$$
\eta=\Phi_{1}+\Phi_{2}-\left(90^{\circ}+\beta-\omega\right)
$$

Now returning to Fig. 16, the transition ray is chosen such that it coincides with plane $A_{2}$ in Fig. 17(a), or the stress discontinuity line $S_{0}$ in Fig. 17(c) if stress discontinuity occurs. Therefore

$$
\psi= \begin{cases}90^{\circ}+\beta-\omega-\Phi_{1} & \text { if } \eta \leqslant 0 \\ \Phi_{2}-\eta_{2} & \text { if } \eta>0\end{cases}
$$

With $\psi$ and $\theta_{0}$ known, two forms of interslice force function, FUN1 and FUN2, are suggested as follows:

$$
\begin{aligned}
& \theta(\xi)= \begin{cases}\theta_{0}+\left(\bar{\delta}-\theta_{0}\right)\left(1-\frac{\xi}{\psi}\right)^{m} & 0 \leqslant \xi \leqslant \psi \\
\theta_{0} & \xi>\psi\end{cases} \\
& \theta(\xi)= \\
& \begin{cases}\theta_{0}+\left(\bar{\delta}-\theta_{0}\right)\left(1-\frac{\xi}{\psi}\right)+0 \cdot 5 m \bar{\phi} \sin \left(1-\frac{\xi}{\psi}\right) \pi & 0 \leqslant \xi \leqslant \psi \\
\theta_{0} & \xi>\psi\end{cases}
\end{aligned}
$$

In more complicated situations, the failure zone may not be divided into two distinct zones, and two other interslice force functions FUN3 and FUN4 are suggested to have the forms of FUN1 and FUN2 respectively within the transition zone:

$$
\begin{gathered}
\theta(\xi)=\theta_{0}+\left(\bar{\delta}-\theta_{0}\right)\left(1-\frac{\xi}{\psi^{\prime}}\right)^{m} \quad \psi^{\prime}=\frac{\pi}{2}+\beta-\omega \quad \text { FUN3 } \\
\theta(\xi)=\theta_{0}+\left(\bar{\delta}-\theta_{0}\right)\left(1-\frac{\xi}{\psi^{\prime}}\right)+0 \cdot 5 m \bar{\phi} \sin \left(1-\frac{\xi}{\psi^{\prime}}\right) \pi \\
\psi^{\prime}=\frac{\pi}{2}+\beta-\omega \text { FUN4 }
\end{gathered}
$$

$m$ in these functions is a non-dimensional parameter, which may be determined by satisfying moment equilibrium condition for the sliding mass.

APPENDIX 2: DERIVATION OF LIMIT EQUILIBRIUM EQUATIONS Choose a typical slice to investigate the equilibrium conditions as shown in Fig. 18. There are five forces acting on the slice:

(a) $W_{k}$, the slice weight, its direction passing through the centroid of the slice. $W_{k}=\gamma_{k} b_{k} h_{k}$, where $b_{k}$ and $h_{k}$ are the width and mean height of slice $k$ respectively

(b) $Q_{k}$, the resultant of the surcharge on the ground surface, its point of action being at the midpoint of the top of the slice. $Q_{k}=$ $q b_{k} \cos \beta / \cos (\beta-\omega)$

(c) $Z_{k-1}$, the interslice force on the upper slice boundary, its inclination with respect to the $x$-axis being $\theta_{k-1}$ and two components $Z^{x}{ }_{k-1}$ and $Z^{y}{ }_{k-1}$

(d) $Z_{k}$, the interslice force on the lower slice boundary, its inclination being $\theta_{k}$; as will be shown, points of application of $Z_{k-1}$ and $Z_{k}$ need not be involved

(e) $R_{k}$, the resultant of the force on the slice base, its inclination with respect to the normal to the slice base being $\bar{\phi}$ and its components $R^{x}{ }_{k}$ and $R^{y}{ }_{k} . R_{k}^{x}=R_{k} \sin \left(\bar{\phi}-\alpha_{k}\right), R_{k}^{y}=R_{k} \cos \left(\bar{\phi}-\alpha_{k}\right)$, where $\alpha_{k}$ is the inclination of the slice base with respect to the $x$-axis. When the slice width, $b_{k}$, is sufficiently small the point of

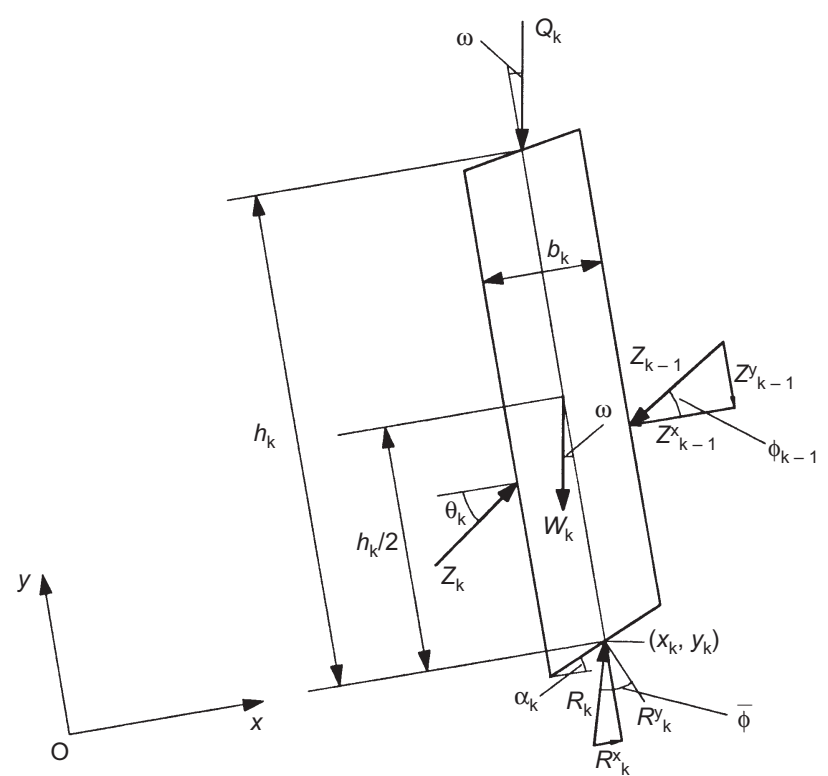

Fig. 18. Forces acting on a typical slice

application of $R_{k}$ can be assumed at the midpoint of the slice base: that is, $\left(x_{k}, y_{k}\right)$ as indicated in Fig. 18.

Considering force equilibrium in the $x$ and $y$ directions results in the following two equations:

$$
\begin{aligned}
& R_{k}^{x}=Z_{k-1} \cos \theta_{k-1}-Z_{k} \cos \theta_{k}+\left(W_{k}+Q_{k}\right) \sin \omega \\
& R_{k}^{y}=Z_{k-1} \sin \theta_{k-1}-Z_{k} \sin \theta_{k}+\left(W_{k}+Q_{k}\right) \cos \omega
\end{aligned}
$$

Recalling the relationship between $R_{k}$ and $R_{k}^{x}, R_{k}^{y}$, solving the above equations simultaneously leads to equations as follows:

$$
\begin{aligned}
Z_{k}= & \frac{1}{\cos \left(\theta_{k}-\alpha_{k}+\bar{\phi}\right)}\left[Z_{k-1} \cos \left(\theta_{k-1}-\alpha_{k}+\bar{\phi}\right)\right. \\
& \left.+\left(Q_{k}+W_{k}\right) \sin \left(\alpha_{k}+\omega-\bar{\phi}\right)\right] \\
Z_{k}= & \frac{1}{\cos \left(\theta_{k}-\alpha_{k}+\bar{\phi}\right)}\left[Z_{k-1}^{x} \cos \left(\alpha_{k}-\bar{\phi}\right)-Z_{k-1}^{y} \sin \left(\alpha_{k}-\bar{\phi}\right)\right. \\
& \left.+\left(Q_{k}+W_{k}\right) \sin \left(\alpha_{k}+\omega-\bar{\phi}\right)\right] \\
R_{k}= & \frac{1}{\cos \left(\theta_{k}-\alpha_{k}+\bar{\phi}\right)}\left[Z_{k-1} \sin \left(\theta_{k-1}-\theta_{k}\right)\right. \\
& \left.+\left(Q_{k}+W_{k}\right) \cos \left(\theta_{k}+\omega\right)\right]
\end{aligned}
$$

Equations (30) represent a recurrence relation between interslice forces, with which the lateral force, $P$, can be obtained by computing interslice forces from the uppermost slice to the last one. Equation (31) is used to check statical admissibility for the slice considered: that is, values of $\alpha_{k}, \theta_{k-1}$ and $\theta_{k}$ should be such that $R_{k}$ is a positive value of a reasonable magnitude in order to avoid numerical difficulties.

Then consider the moment equilibrium condition for the overall sliding mass. Because interslice forces, $Z_{k}, k=1,2, \ldots, n-1$, cancel out, they may be excluded from consideration for the overall moment equilibrium. For slice $k$, taking moments about $\mathrm{O}$ of forces $R_{k}^{x}, R^{y}{ }_{k}, W_{k}$ and $Q_{k}$, the moment being taken as positive when it produces an anti-clockwise rotation, the total contribution of these forces is

$$
\begin{aligned}
M^{(k)}(\mathrm{O})= & -R_{k}^{x} y_{k}+R_{k}^{y} x_{k}-\left(W_{k}+Q_{k}\right) \cos \omega x_{k} \\
& +W_{k} \sin \omega\left(y_{k}+h_{k} / 2\right)+Q_{k} \sin \omega\left(y_{k}+h_{k}\right)
\end{aligned}
$$

Substituting $R^{x}{ }_{k}$ and $R^{y}{ }_{k}$ from equation (29) and rearranging:

$$
\begin{aligned}
M^{(k)}(\mathrm{O})= & Z_{\mathrm{k}}\left(y_{k} \cos \theta_{k}-x_{k} \sin \theta_{k}\right) \\
& +Z_{k-1}\left(-y_{k} \cos \theta_{k-1}+x_{k} \sin \theta_{k-1}\right) \\
& +W_{k} \sin \omega \frac{h_{k}}{2}+Q_{k} \sin \omega \cdot h_{k}
\end{aligned}
$$


Summing $M^{(k)}(\mathrm{O})$ for all slices and rearranging:

$$
\begin{aligned}
\sum_{k=1}^{n} M^{(k)}(\mathrm{O})= & \left(y_{n} \cos \theta_{n}-x_{n} \sin \theta_{n}\right) \cdot Z_{n} \\
& +\sum_{k=1}^{n-1}\left[\left(y_{k}-y_{k+1}\right) \cos \theta_{k}-\left(x_{k}-x_{k+1}\right) \sin \theta_{k}\right] \cdot Z_{k} \\
& +\sum_{k=1}^{n}\left(W_{k} \sin \omega \frac{h_{k}}{2}+Q_{k} \sin \omega \cdot h_{k}\right)
\end{aligned}
$$

Noting the following geometrical relations:

$$
\begin{aligned}
& x_{k}-x_{k+1}=\frac{b_{k}+b_{k+1}}{2} \\
& y_{k}-y_{k+1}=\frac{b_{k}}{2} \tan \alpha_{k}+\frac{b_{k+1}}{2} \tan \alpha_{k+1}
\end{aligned}
$$

and $Z_{0}=0$, equation (33) becomes

$$
\begin{aligned}
\sum_{k=1}^{n} M^{(k)}(\mathrm{O})= & Z_{n} \frac{b_{n}}{2} \frac{\sin \left(\alpha_{n}-\theta_{n}\right)}{\cos \alpha_{n}} \\
& +\sum_{k=1}^{n-1}\left[\frac{\sin \left(\alpha_{k}-\theta_{k}\right)}{\cos \alpha_{k}} \cdot \frac{b_{k}}{2}+\frac{\sin \left(\alpha_{k+1}-\theta_{k}\right)}{\cos \alpha_{k+1}} \cdot \frac{b_{k+1}}{2}\right] \cdot Z_{k} \\
& +\sum_{k=1}^{n}\left(W_{k} \sin \omega \frac{h_{k}}{2}+Q_{k} \sin \omega \cdot h_{k}\right)
\end{aligned}
$$

Equation (34) may be rewritten in a form of recurrence relation like equation (30a):

$$
\begin{aligned}
M_{k}= & M_{k-1}+\left[\frac{\sin \left(\alpha_{k}-\theta_{k}\right)}{\cos \alpha_{k}} \cdot \frac{b_{k}}{2}+\frac{\sin \left(\alpha_{k+1}-\theta_{k}\right)}{\cos \alpha_{k+1}} \cdot \frac{b_{k+1}}{2}\right] \cdot Z_{k} \\
& +W_{k} \sin \omega \frac{h_{k}}{2}+Q_{k} \sin \omega \cdot h_{k}
\end{aligned}
$$

where $k=1,2, \ldots, n ; b_{n+1}=0 ; M_{0}=0 ; M_{n}=\sum_{k=1}^{n} M^{(k)}(\mathrm{O}) . M_{k}$ is defined as the interslice moment.

\section{APPENDIX 3. SOLVING FOR PARAMETER $m$ BY USE OF THE} NEWTON-RAPHSON METHOD

$M_{k}, Z_{k}, k=1,2, \ldots, n$, in equations (1) and equation (2) may be thought of as functions of the parameter $m$ in the interslice force function. The problem of determination of the value of $m$ is essentially the solution of the one-variable non-linear equation (equation (4)). The solution can be approximated successively starting from an appropriate initial value of $m$, denoted as $m_{0}$. In general, assuming the value of $m$ at the $s$ th stage is obtained as $m_{s}$, the next value, $m_{s+1}$, is determined using the following equation:

$$
m_{s+1}=m_{s}-\frac{M_{n}\left(m_{s}\right)+C_{1} Z_{n}\left(m_{s}\right)+C_{2}}{\frac{\mathrm{d} M_{n}}{\mathrm{~d} m}\left(m_{s}\right)+C_{1} \frac{\mathrm{d} Z_{n}}{\mathrm{~d} m}\left(m_{s}\right)}
$$

The derivatives $\mathrm{d} M_{n} / \mathrm{d} m, \mathrm{~d} Z_{n} / \mathrm{d} m$ can be obtained using the following recurrence equations, which are readily derived by differentiating equations (1) and (2) respectively with respect to $m$ :

$$
\begin{aligned}
\frac{\mathrm{d} Z_{k}}{\mathrm{~d} m}= & \frac{1}{\cos \left(\theta_{k}-\alpha_{k}+\bar{\phi}\right)}\left[\cos \left(\theta_{k-1}-\alpha_{k}+\bar{\phi}\right) \frac{\mathrm{d} Z_{k-1}}{\mathrm{~d} m}\right. \\
& -\sin \left(\theta_{k-1}-\alpha_{k}+\bar{\phi}\right) Z_{k-1} \frac{\mathrm{d} \theta_{k-1}}{\mathrm{~d} m} \\
& \left.+\sin \left(\theta_{k}-\alpha_{k}+\bar{\phi}\right) Z_{k} \frac{\mathrm{d} \theta_{k}}{\mathrm{~d} m}\right] \\
\frac{\mathrm{d} M_{k}}{\mathrm{~d} m}= & \frac{\mathrm{d} M_{k-1}}{\mathrm{~d} m} \\
& +\left[\frac{\sin \left(\alpha_{k}-\theta_{k}\right)}{\cos \alpha_{k}} \cdot \frac{b_{k}}{2}+\frac{\sin \left(\alpha_{k+1}-\theta_{k}\right)}{\cos \alpha_{k+1}} \cdot \frac{b_{k+1}}{2}\right] \cdot \frac{\mathrm{d} Z_{k}}{\mathrm{~d} m} \\
& -\left[\frac{\cos \left(\alpha_{k}-\theta_{k}\right)}{\cos \alpha_{k}} \cdot \frac{b_{k}}{2}+\frac{\cos \left(\alpha_{k+1}-\theta_{k}\right)}{\cos \alpha_{k+1}} \cdot \frac{b_{k+1}}{2}\right] Z_{k} \frac{\mathrm{d} \theta_{k}}{\mathrm{~d} m}
\end{aligned}
$$

where $\mathrm{d} \theta_{k-1} / \mathrm{d} m, \mathrm{~d} \theta_{k} / \mathrm{d} m$ can be calculated if the interslice force function is specified.

\section{REFERENCES}

Baker, R. (1980). Determination of the critical slip surface in slope stability computations. Int. J. Numer. Anal. Methods Geomech. 4, 333-359.

Bellman, R. (1957). Dynamic programming. Princeton, NJ: Princeton University Press.

Caquot, A. \& Kerisel, J. (1948). Tables for the calculation of passive pressure, active pressure and bearing capacity of foundations. Paris: Gauthier-Villars.

Chang, M. F. (1997). Lateral earth pressures behind rotating walls. Can. Geotech. J. 34, 498-509.

Chen, W. F. (1975). Limit analysis and soil plasticity. Amsterdam: Elsevier.

Chen, W. F. \& Liu, X. L. (1990). Limit analysis in soil mechanics. New York: Elsevier.

Fang, Y. S. \& Ishibashi, I. (1986). Static earth pressures with various wall movements. J. Geotech. Engng. Div., ASCE 112, No. 3, 317333

Fang, Y. S., Cheng, F. P., Chen, R. C. \& Fan, C. C. (1993). Earth pressures under general wall movements. Geotech. Engng 24, No. 2, $113-131$.

Graham, J. (1968). Plane plastic failure in cohesionless soils. Géotechnique 18, No. 3, 301-306.

Hettiarachi, D. R. P. \& Reece, A. R. (1974). The calculation of passive soil resistance. Géotechnique 24, No. 3, 289-310.

James, R. G. \& Bransby, P. L. (1970). Experimental and theoretical investigations of a passive pressure problem. Géotechnique 20, No. $1,17-37$.

Janbu, N. (1957). Earth pressure and bearing capacity calculations by generalised procedure of slices. Proc. 4th Int. Conf. Soil Mech. Found. Engng, London 2, 207-212

Krey, H. (1936). Erddruck, Erdwidestant und Tragfahigkeit des Baugrunds, 5th edn. Berlin: Ernst.

Lee, I. K. \& Herington, J. R. (1972). A theoretical study of the pressures acting on a rigid wall by a sloping earth or rock fill. Géotechnique 22, No. 1, 1-26

Morgenstern, N. R. \& Price, V. E. (1965). The analysis of stability of general slip surfaces. Géotechnique 15, No. 1, 79-93.

Powrie, W. (1997). Soil mechanics: concepts and applications. London: E \& FN Spon.

Rahardio, H. \& Fredlund, D. G. (1983). General limit equilibrium method for lateral earth force. Can. Geotech. J. 21, 166-175.

Shields, D. H. \& Tolunay, A. Z. (1973). Passive pressure coefficients by method of slices. J. Soil Mech. Found. Div., ASCE 99 No. SM12, $1043-1053$.

Sokolovski, V. V. (1965). Statics of granular media. New York: Pergamon Press.

Terzaghi, K. (1943). Theoretical soil mechanics. New York: John Wiley \& Sons.

Zhu, D.-Y. (1997). Critical slip field and its numerical simulation. Chin. J. Geotech. Engng., 19, No. 1,63-69.

Zhu, D.-Y. (1999). A method of soil stability analysis: method of critical slip field. $\mathrm{PhD}$ thesis, Nanjing Engineering Institute. 